\title{
ANÁLISIS DE LAS ELECCIONES AUTONÓMICAS Y MUNICIPALES DE 26 DE MAYO DE 1991
}

CARMEN FERNÁNDEZ-MIRANDA CAMPOAMOR

Profesora Titular de Derecho Constitucional

UNED 


\section{SUMARIO}

I. Campaña electoral.- II. Datos electorales. - 1. Diputados autonómicos electos en las 13 Comunidades Autónomas. 2. Concejales electos en las capitales de provincia. III. ANALISIS GENERAL DE LOS RESULTADOS. - IV. PACTOS POSTELECTORALES.V. ANALISIS DE LOS RESULTADOS AUTONOMICOS EN LAS 13 COMUNIDADES AUTÓNOMAS. - VI. TRASCENDENCIA DE LAS ELECCIONES AUTONÓMICAS en la COMPOSICION DEL SENAdO. - VII. Conclusiones. 


\section{Siglas utilizadas}

AIC: Agrupación Independiente Canaria

AHI: Agrupación Herrereña Independiente

AM: Asamblea Majorera

ATI: $\quad$ Agrupación Tinerfeña Independiente

CDS: Centro Democrático y Social

CiU: Convergencia i Unió

CNG: Coalición Nacionalista Gallega

EA: Eusko Alkartasuna

EE: $\quad$ Euskadiko Ezquerra

EEM: $\quad$ Entesa d'Ezquerra de Menorca

EU: $\quad$ Extremadura Unida

FIEF: Federació d'Independents d'Eivissa i Formentera

HB: Herri Batasuna

IC-IU: Iniciativa Canaria-Izquierda Unida

IU: Izquierda Unida. Coalición

PA: Partido Andalucista

PAR: $\quad$ Partido Aragonés Regionalista

PAS-UNA: Coalición del Partido Asturianista y Unidad Nacionalista Astur PCU: $\quad$ Partido de Ceuta Unida

PFPC: $\quad$ Partido para el Progreso y Futuro de Ceuta

PNV: Partido Nacionalista Vasco

PP: $\quad$ Partido Popular

PR: $\quad$ Partido Riojano

PRC: Partido Regionalista de Cantabria

PSM: Partido Socialista de Mallorca

PSOE: Partido Socialista Obrero Español

UIM: Unió d'Independents de Mallorca

UM: Unión Mallorquina

UPCA: Unión para el Progreso de Cantabria

UV: $\quad$ Unión Valenciana

UPN: Unión del Pueblo Navarro 

Revista de Derecho Político, núm. 35, 1992, pp. 239-328

\title{
ANÁLISIS DE LAS ELECCIONES AUTONÓMICAS Y MUNICIPALES DE 26 DE MAYO DE 1991
}

POA

\author{
CARMEN FERNANDEZ-MIRANDA CAMPOAMOR
}

Profesora Titular de Derecho Constitucional

UNED

\section{CAMPAÑA ELECTORAL}

El 26 de mayo de 1991 se celebran elecciones municipales en las 17 Comunidades Autónomas para elegir las Corporaciones Municipales de más de 8.000 Ayuntamientos. A su vez, se celebran elecciones a las Asambleas Legislativas de 13 Comunidades Autónomas (salvo Andalucía, Cataluña, Galicia y País Vasco) ${ }^{1}$. Hay que destacar la incorporación al censo electoral, para esta convocatoria, de más de un millón de vontantes de dieciocho años.

La nota distintiva de este tipo de elecciones que afectan directamente a la calidad de vida del ciudadano es la mayor valoración de la eficacia en la gestión que de las ideologías partidistas. Junto a ello es necesario resaltar la importancia esencial de los partidos nacionalistas y regionalis-

1 Las elecciones municipales son convocadas por R. D. 391/1991 de 1 de abril (BOE 2 de abril de 1991).

El BOE de 2 de abril, publica también los Decretos de la Presidericia de las $13 \mathrm{Co}$ munidades Autónomas por los que se convocan elecciones a las respectivas Asambleas Legislativas.

Posteriormente, el R. D. 1334/1991 de 9 de septiembre (BOE de 10 de septiembre de 1991) convoca elecciones parciales, en el plazo de seis meses, en aquellas circunscripciones en que no se hubieran presentado candidaturas en las elecciones locales de 26 de mayo y en aquellas que por sentencia firme o por Acuerdo de la Junta Electoral competente se ha declarado su nulidad total o parcial. 
tas con programas y candidatos más conocidos del votante; ello contribuye a que se personalice más la elección ${ }^{2}$.

Esta campaña concreta se caracteriza, en general, por el aburrimiento, la ausencia de debates ideológicos y el pragmatismo de los partidos ante un elector impresionado por varias circunstancias concurrentes; entre ellas, las acusaciones de corrupción en determinados sectores políticos, el derrumbamiento de los regímenes de los países del Este y la fecha de 1992 en que se celebrarán tres acontecimientos de importante significación para nuestro pais: Ia Exposición Universal de Sevilla, los Juegos Olímpicos de Barcelona y la capitalidad cultural europea de Madrid. Todo ello coadyuva a dar una relevancia especial a estos comicios que se consideran un ensayo de cara a las legislativas próximas ${ }^{3}$.

Se observa en esta campaña un intento del PP por aprovechar el desgaste del PSOE en la gestión municipal de las grandes ciudades, pretendiendo conquistar el voto de las clases medias urbanas con la promesa de una gestión eficaz que logre detener el deterioro - cada vez más evidente - de la convivencia ciudadana, asfixiada por la droga - con su terrible secuela de delincuencia y la consiguiente inseguridad ciudadana-, la contaminación y los graves problemas de circulación que dificultan la calidad de vida. Es preciso señalar los numerosos movimientos sociales generados por estos problemas, que piden respuestas inmediatas al margen de los partidos políticos con representación parlamentaria.

Por su parte, IU aprovecha ese mismo desgaste del partido gobernante para presentar un proyecto de regeneración que aglutine aquellos sectores de la izquierda desencantados ante las continuas acusaciones de corrupción politica. Tanto el líder del PP - José M. ${ }^{a}$ Aznar-como el de IU - Julio Anguita- buscan consolidar su liderazgo al frente de unas formaciones que han sufrido importantes modificaciones en busca de un espacio electoral más amplio. Para el partido de Adolfo Suárez - CDS-, el lograr buenos resultados en esta oportunidad, es imprescindible para pervivir.

Otro dato a considerar en esta campaña es la oferta de alianzas post-electorales, en las que el CDS parece decantarse hacia el PSOE - quien evita hacerlo blanco de sus críticas- lo mismo que IU, capaz de apoyar al partido gobernante a fin de evitar el dominio de la derecha, pero rechazando todo pacto global de gobierno. A su vez, el PP cuida especiaimente sus relaciones con los partidos regionalistas, posibles socios futuros. 1991.

2 A. TORRES DEL MORAL: “La representación política», en Derecho Político I, UNED,

3 Ver El País de 12 y 28 de mayo de 1991. 
Como colofón de todos estos datos, puede decirse que el dia 26 de mayo el elector se plantea más el evitar que acceda al gobierno municipal o autonómico éste o aquel partido, que el optar directamente por un determinado programa.

\section{DATOS ELECTORALES}

\section{Diputados autonómicos electos en las $\mathbf{1 3}$ Comunidades Autónomas ${ }^{4}$}

Cinco meses después de haberse celebrado las elecciones autonómicas, el $B O E$ no ha publicado los resultados y la Junta Electoral Central sólo dispone de los enviados por cuatro Comunidades Autónomas: Navarra, Canarias, Madrid y La Rioja; algunas de las restantes los han publicado en su Boletín Oficial y otras no han hecho ningún tipo de publicación. En consecuencia, no existe ningún tipo de control estatal sobre los resultados autonómicos; cada Autonomía ha tomado una decisión individual sin que la Junta Electoral Central, en cinco meses, haya hecho nada para unificar criterios; se podría decir - parafraseando al profesor M. Gonzalo- que esta cuestión, si bien no es secreta, entra dentro de lo misterioso. Junto a los datos electorales de esta convocatoria se aportan resultados de otras elecciones a fin de tener una visión más completa de la evolución de cada partido.

4 La Ley Electoral, en su artículo 108.4, establece que: «... la Junta archivará uno de los tres ejemplares del acta, remitirá el segundo a la Cámara o Corporación de la que vayan a formar parte los electos y el tercero a la Junta Electoral Central que, en el período de cuarenta días, procederá a la publicación en el Boletín Oficial del Estado de los resultados generales y por circunscripciones, sin perjuicio de los recursos contencioso-electorales contra la proclamación de electos". 


\section{ARAGON}

\section{(ELECCIONES A CORTES DE ARAGON)}

Resultados publicados en $B O A, n .^{\circ} 80,1$ julio 1991

\begin{tabular}{lr}
\hline Diputados: & 67 \\
Censo: & 959.596 \\
Votantes: & 617.848 \\
Votos válidos: & 613.545 \\
Votos nulos: & 4.303 \\
Votos en blanco: & 7.981 \\
\hline
\end{tabular}

\begin{tabular}{|l|c|c|c|}
\hline \multirow{2}{*}{ Partidos } & 1991 & 1987 & 1983 \\
\cline { 2 - 4 } & Escaños & Escaños & Escaños \\
\hline PSOE & 30 & 27 & 33 \\
PAR & 17 & 19 & 13 \\
PP & 17 & 13 & 18 \\
IU & 3 & 2 & 1 \\
CDS & - & 6 & 1 \\
\hline
\end{tabular}

\begin{tabular}{|lr|}
\hline \multicolumn{2}{|c|}{ N. ${ }^{\circ}$ OE VOTOS 1991} \\
PSOE & 247.485 \\
PAR & 151.420 \\
PP & 126.892 \\
IU & 41.367 \\
CDS & 18.929 \\
\hline
\end{tabular}




\section{ASTURIAS}

(ELECCIONES A LA JUNTA GENERAL DEL PRINCIPADO)

Resultados suministrados por el Principado de Asturias

\begin{tabular}{lr} 
Diputados: & 45 \\
Censo: & 901.041 \\
Votantes: & 532.424 \\
Votos a candidaturas: & 521.907 \\
Votos nulos: & 4.024 \\
Votos en blanco: & 6.493 \\
\hline
\end{tabular}

\begin{tabular}{|l|c|c|c|}
\hline \multirow{2}{*}{ PARTIDOS } & 1991 & 1987 & 1983 \\
\cline { 2 - 4 } & Escaños & EsCANOS & ESCANOS \\
\hline PSOE & 21 & 20 & 26 \\
PP & 15 & 13 & 14 \\
CDS & 2 & 8 & - \\
PAS-UNA & 1 & - & - \\
IU & 6 & 4 & 5 \\
\hline
\end{tabular}

\begin{tabular}{|lr|}
\hline \multicolumn{2}{|c|}{ N. $^{\circ}$ DE VOTOS 1991} \\
PSOE & 215.950 \\
PP & 160.645 \\
IU & 78.551 \\
CDS & 36.013 \\
PAS-UNA & 14.497 \\
\hline
\end{tabular}


BALEARES

(ELECCIONES AL PARLAMENT DE LES ILLES BALEARS)

Resultados publicados en BOCAIB, n. 84 , de 2 julio 1991

\begin{tabular}{lr}
\hline Diputados: & 59 \\
Censo: & 566.782 \\
Votantes: & 341.298 \\
Votos a candidaturas: & 335.758 \\
Votos válidos: & 339.192 \\
Votos nulos: & 2.106 \\
Votos en blanco: & 2.934 \\
\hline
\end{tabular}

\begin{tabular}{|l|c|c|c|}
\hline \multirow{2}{*}{ PARTIDOS } & 1991 & 1987 & 1983 \\
\cline { 2 - 4 } & ESCANOS & ESCANOOS & ESCANOS \\
\hline PSOE & 21 & 21 & 21 \\
PP-UM & 31 & 25 & 21 \\
CDS & - & 5 & - \\
EEM & 2 & - & - \\
PSM & 3 & 4 & 6 \\
FIEF & 1 & faltan datos & faltan datos \\
UIM & 1 & - & - \\
\hline
\end{tabular}

\begin{tabular}{|lr|}
\hline \multicolumn{2}{|c|}{ N. $^{\circ}$ DE VOTOS 1991} \\
PSOE & 102.060 \\
PP-UM & 160.512 \\
CDS & 9.938 \\
EEM & 4.654 \\
PSM & 22.522 \\
FIEF & 2.468 \\
\hline
\end{tabular}




\section{CANTABRIA}

(ELECCIONES A LA ASAMBLEA)

Datos oficiales proporcionados por esta Comunidad

\begin{tabular}{lr}
\hline Diputados: & 39 \\
Censo: & 409.390 \\
Votantes: & 297.795 \\
Votos nulos: & 2.542 \\
Votos en blanco: & 5.018 \\
\hline
\end{tabular}

\begin{tabular}{|l|c|c|c|}
\hline \multirow{2}{*}{ PARTIOOS } & 1991 & 1987 & 1983 \\
\cline { 2 - 4 } & Escaños & Escaños & EsCAÑOS \\
\hline PSOE & 16 & 13 & 15 \\
PP & 6 & 19 & 18 \\
CDS & - & 3 & - \\
IU & - & - & - \\
PRC & 2 & 5 & 2 \\
UPCA & 15 & - & - \\
\hline
\end{tabular}

\begin{tabular}{|lr|}
\hline \multicolumn{2}{|c|}{ \% DE VOTOS 1991 } \\
PSOE & 34,73 \\
PP & 14,39 \\
CDS & 2,69 \\
IU & 4,35 \\
PRC & 6,46 \\
UPCA & 33,62 \\
\hline
\end{tabular}




\section{CANARIAS}

(PARLAMENTO DE CANARIAS)

Datos llegados a la Junta Electoral Central el 10 de junio de 1991 y suministrados por ésta

\begin{tabular}{lrr}
\hline $\begin{array}{l}\text { N. }{ }^{\circ} \text { de electores censados: } \\
\text { N. }{ }^{\circ} \text { de certificaciones censales aportadas: }\end{array}$ & 1.135 .550 \\
& & 1.397 \\
& TOTAL (N. ${ }^{\circ}$ electores): & 1.136 .947 \\
\hline Diputados: & 60 & \\
Votantes: & 702.300 & \\
Votos a candidaturas: & 691.897 \\
Votos válidos: & 697.220 \\
Votos nulos: & 5.080 \\
Votos en blanco: & 5.323 \\
\hline
\end{tabular}

\begin{tabular}{|l|c|c|c|}
\hline \multirow{2}{*}{ Partidos } & 1991 & 1987 & 1983 \\
\cline { 2 - 4 } & ESCANOS & ESCANOS & ESCANOS \\
\hline PSOE & 23 & 21 & 27 \\
PP & 6 & 6 & 17 \\
CDS & 7 & 13 & 6 \\
ICAN & 5 & 2 & 1 \\
AIC & 16 & 11 & - \\
AM & 2 & 3 & faltan datos \\
AHI & 1 & 2 & faltan datos \\
\hline
\end{tabular}

\begin{tabular}{|lr|}
\hline \multicolumn{2}{|c|}{ N. $^{\circ}$ DE VOTOS 1991} \\
PSOE & 229.690 \\
PP & 89.251 \\
ICAN & 85.015 \\
CDS & 100.197 \\
AIC & 157.859 \\
AM & 4.906 \\
AHI & 1.485 \\
\hline
\end{tabular}




\section{CASTILLA-LEON}

\section{(ELECCIONES A CORTES DE CASTILLA-LE்ON)}

Datos publicados en $B O C$ y $L, n .^{\circ} 126,3$ julio 1991

\begin{tabular}{lr}
\hline Diputados: & 84 \\
N. ${ }^{\circ}$ electores: & 2.069 .469 \\
Votos válidos: & 1.385 .207 \\
Votos nulos: & 13.031 \\
Votos en blanco: & 23.028 \\
\hline
\end{tabular}

\begin{tabular}{|l|c|c|c|}
\hline \multirow{2}{*}{ PARTIDOS } & 1991 & 1987 & 1983 \\
\cline { 2 - 4 } & Escaños & EsCANOS & Escaños \\
\hline PP & 43 & 32 & 39 \\
PSOE & 35 & 32 & 42 \\
CDS & 5 & 18 & 2 \\
IU & 1 & - & - \\
\hline
\end{tabular}

N. ${ }^{\circ}$ DE VOTOS 1991

PP $\quad 602.773$

PSOE $\quad 504.709$

CDS $\quad 69.212$

IU $\quad 20.506$ 


\section{CASTILLA-LA MANCHA}

(ELECCIONES A LA ASAMBLEA)

Diputados:

47

N. ${ }^{\circ}$ electores:

1.296 .625

N. ${ }^{\circ}$ votantes:

944.970

\begin{tabular}{|l|c|c|c|}
\hline \multirow{2}{*}{ PARTIDOS } & 1991 & 1987 & 1983 \\
\cline { 2 - 4 } & EsCAÑOS & EsCAÑOS & ESCAÑOS \\
\hline PSOE & 27 & 25 & 23 \\
PP & 19 & 18 & 21 \\
IU & 1 & - & - \\
CDS & - & 4 & - \\
\hline
\end{tabular}

\begin{tabular}{|lc|}
\hline \multicolumn{2}{|c|}{ \% DE VOTOS 1991} \\
PSOE & 52,17 \\
PP & 35,91 \\
CDS & 3,49 \\
IU & 6,16 \\
\hline
\end{tabular}




\section{EXTREMADURA}

(ELECCIONES A LA ASAMBLEA)

Datos publicados en $D O E$, n. $^{\circ} 45,13$ junio 1991

\begin{tabular}{lr} 
Diputados: & 65 \\
Censo: & 824.866 \\
Votantes: & 584.378 \\
Votos válidos: & 580.511 \\
Votos nulos: & 3.867 \\
Votos en blanco: & 4.508 \\
\hline
\end{tabular}

\begin{tabular}{|l|c|c|c|}
\hline \multirow{3}{*}{ PartioOS } & 1991 & 1987 & 1983 \\
\cline { 2 - 4 } & Escaños & Escaños & Escaños \\
\hline PSOE & 39 & 34 & 36 \\
PP & 19 & 17 & 20 \\
CDS & 3 & 8 & - \\
IU & 4 & 2 & 4 \\
EU & - & 4 & 6 \\
\hline
\end{tabular}

\begin{tabular}{|lr|}
\hline \multicolumn{2}{|c|}{ N. ${ }^{\circ}$ DE VOTOS 1991} \\
PSOE & 335.809 \\
PP & 155.485 \\
CDS & 33.291 \\
IU & 41.290 \\
EU & 14.503 \\
\hline
\end{tabular}




\section{MADRID}

\section{(ELECCIONES A LA ASAMBLEA DE MADRID)}

Datos enviados a la Junta Electoral Central el 6 de junio de 1991 y suministrados por ésta

\begin{tabular}{lr} 
Diputados: & 101 \\
Censo: & 3.837 .680 \\
Votantes: & 2.251 .613 \\
Votos a candidaturas: & 2.213 .472 \\
Votos válidos: & 2.242 .344 \\
Votos nulos: & 7.269 \\
Votos en blanco: & 28.872 \\
\hline
\end{tabular}

\begin{tabular}{|l|c|c|c|}
\hline \multirow{2}{*}{ PARTIDOS } & 1991 & 1987 & 1983 \\
\cline { 2 - 4 } & Escaños & Escaños & EsCaños \\
\hline PP & 47 & 32 & - \\
PSOE & 41 & 40 & - \\
IU & 13 & 7 & - \\
CDS & - & 17 & - \\
\hline
\end{tabular}

\begin{tabular}{|lr|}
\hline \multicolumn{2}{|c|}{ N. ${ }^{\circ}$ DE VOTOS 1991} \\
PP & 956.765 \\
PSOE & 820.510 \\
IU & 270.558 \\
CDS & 75.081 \\
\hline
\end{tabular}




\section{MURCIA}

(ELECCIONES A LA ASAMBLEA REGIONAL)

Datos oficiales de la Junta Electoral provincial de Murcia)

\begin{tabular}{lr}
\hline Diputados: & 45 \\
Censo: & 778.256 \\
Votantes: & 522.802 \\
Votos a candidaturas: & 512.928 \\
Votos válidos: & 517.774 \\
Votos nulos: & 5.028 \\
Votos en blanco: & 4.846 \\
\hline
\end{tabular}

\begin{tabular}{|l|c|c|c|}
\hline \multirow{2}{*}{ PARTIDOS } & 1991 & 1987 & 1983 \\
\cline { 2 - 4 } & Escaños & Escaños & Escaños \\
\hline PSOE & 24 & 25 & 26 \\
PP & 17 & 16 & 16 \\
CDS & - & 3 & - \\
IU & 4 & 1 & 1 \\
\hline
\end{tabular}

\begin{tabular}{|lr|}
\hline \multicolumn{2}{|c|}{ N. ${ }^{\circ}$ DE VOTOS 1991} \\
PSOE & 234.421 \\
PP & 173.491 \\
CDS & 25.940 \\
IU & 52.593 \\
\hline
\end{tabular}


NAVARRA

(ELECCIONES AL PARLAMENTO DE NAVARRA)

Datos enviados a la Junta Electoral Central el 31 de mayo de 1991 y suministrados por ésta. BO de Navarra de 21 de julio de 1991

\begin{tabular}{lr}
\hline Diputados: & 50 \\
Censo: & 414.913 \\
Votantes: & 276.773 \\
Votos a candidaturas: & 271.045 \\
Votos válidos: & 274.682 \\
Votos nulos: & 2.091 \\
Votos en blanco: & 3.637 \\
\hline
\end{tabular}

\begin{tabular}{|l|c|c|c|}
\hline \multirow{2}{*}{ PARTIOOS } & 1991 & 1987 & 1983 \\
\cline { 2 - 4 } & ESCAÑOS & ESCAÑOS & ESCAÑOS \\
\hline PSN-PSOE & 19 & 15 & 20 \\
EA & 3 & 4 & - \\
PP-UPN & 20 & 2 & 8 \\
PNV & - & - & 3 \\
IU & 2 & - & - \\
CDS & - & 4 & - \\
UPN & - & 14 & 13 \\
HB & 6 & 7 & 6 \\
\hline
\end{tabular}

\begin{tabular}{|lr|}
\hline \multicolumn{2}{|c|}{ N. $^{\circ}$ DE VOTOS 1991} \\
PSOE & 91.645 \\
PP-UPN & 96.055 \\
CDS & 5.650 \\
HB & 30.762 \\
EE & 5.824 \\
EA & 15.170 \\
IU & $11: 167$ \\
\hline
\end{tabular}




\section{LA RIOJA}

\section{(ELECCIONES A LA DIPUTACION GENERAL)}

Datos enviados a la Junta Electoral Central el 7 de junio de 1991* y publicados en BOLR el 13 de junio de 1991

\begin{tabular}{lr} 
Diputados: & 33 \\
Censo: & 210.080 \\
Votantes: & 144.729 \\
Votos a candidaturas: & 141.220 \\
Votos válidos: & 143.593 \\
Votos nulos: & 1.136 \\
Votos en blanco: & 2.373 \\
\hline
\end{tabular}

\begin{tabular}{|l|c|c|c|}
\hline \multirow{2}{*}{ Partidos } & 1991 & 1987 & 1983 \\
\cline { 2 - 4 } & Escaños & Escaños & Escaños \\
\hline PSOE & 16 & 14 & 18 \\
PP & 15 & 13 & 15 \\
CDS & - & 4 & - \\
IU & - & - & - \\
PR & 2 & 2 & 2 \\
\hline
\end{tabular}

\begin{tabular}{|lr|}
\hline \multicolumn{2}{|c|}{ N. ${ }^{\circ}$ DE Votos 1991} \\
PSOE & 60.843 \\
PP & 59.876 \\
IU & 6.499 \\
PR & 7.731 \\
CDS & 6.271 \\
\hline
\end{tabular}




\section{COMUNIDAD VALENCIANA}

\section{(ELECCIONES A CORTES DE VALENCIA)}

Resultados oficiales

\begin{tabular}{lc}
\hline Diputados: & 89 \\
\hline Censo: & 2.908 .853 \\
Votantes: & 2.015 .804 \\
\hline
\end{tabular}

\begin{tabular}{|l|c|c|c|}
\hline \multirow{2}{*}{ PARTIDOS } & 1991 & 1987 & 1983 \\
\cline { 2 - 4 } & ESCAÑOS & ESCANOS & ESCAÑOS \\
\hline PSOE & 45 & 42 & 51 \\
PP & 31 & 24 & 32 \\
UV & 6 & 6 & 6 \\
IU & 7 & 6 & - \\
CDS & - & 11 & - \\
\hline
\end{tabular}

\begin{tabular}{|lr|}
\hline \multicolumn{2}{|c|}{ N. ${ }^{\circ}$ DE VOTOS 1991} \\
PSOE & 858.022 \\
PP & 558.462 \\
UV & 207.561 \\
IU & 150.840 \\
CDS & 76.249 \\
\hline
\end{tabular}


2. Concejales electos en las capitales de provincia ${ }^{5}$

PROVINCIA:

ÁLAVA

J. ELECTORAL DE ZONA: VITORIA

MUNICIPIO: VITORIA-GASTEIZ

\begin{tabular}{lr}
\hline N. ${ }^{\circ}$ electores: & 159.369 \\
N. ${ }^{\circ}$ votantes: & 85.815 \\
N. ${ }^{\circ}$ votos de candidatura: & 84.303 \\
N. ${ }^{\circ}$ votos en blanco: & 837 \\
N..$^{\circ}$ votos válidos: & 85.140 \\
N..$^{\circ}$ votos nulos: & 675 \\
\hline
\end{tabular}

\begin{tabular}{|l|c|c|}
\hline CANDIDATURAS & $\begin{array}{c}\text { VotOS } \\
\text { OBTENIDOS }\end{array}$ & $\begin{array}{c}\text { N. CONCEJ. } \\
\text { ELECTOS }\end{array}$ \\
\hline EE & 3.553 & 0 \\
IU-EB & 1.159 & 0 \\
UNIDAD ALAVESA & 18.749 & 7 \\
EAJ-PNV & 22.430 & 8 \\
PSE-PSOE & 15.986 & 6 \\
CDS & 1.667 & 0 \\
PP & 7.061 & 2 \\
HB & 7.779 & 3 \\
EHB & 310 & 0 \\
EA & 4.542 & 1 \\
UVE & 508 & 0 \\
COALICION ALIANZA POR LA & 114 & 0 \\
REPÚBLICA & 445 & 0 \\
JV & & \\
\hline
\end{tabular}

5 BOE núm. 173. Suplemento, 20 de julio de 1991. 
PROVINCIA:

ALBACETE

J. ELECTORAL DE ZONA: ALBACETE

MUNICIPIO:

ALBACETE
N. ${ }^{\circ}$ electores:
94.090
N. ${ }^{\circ}$ votantes:
55.051
N. ${ }^{\circ}$ votos de candidatura:
53.626
N. ${ }^{\circ}$ votos en blanco:
928
N. ${ }^{\circ}$ votos válidos:
54.554
N. ${ }^{\circ}$ votos nulos:
497

\begin{tabular}{|l|c|c|}
\hline CANDIDATURAS & $\begin{array}{c}\text { VoTOS } \\
\text { OBTENIDOS }\end{array}$ & $\begin{array}{c}\text { N. }{ }^{\circ} \text { CONCEJ. } \\
\text { ELECTOS }\end{array}$ \\
\hline $\begin{array}{l}\text { COALICION PLATAFORMA DE } \\
\text { IZQUIERDA }\end{array}$ & 205 & 0 \\
PSOE & 25.957 & 14 \\
CDS & 1.950 & 0 \\
IU & 5.678 & 3 \\
PARTIDO REGIONALISTA & 596 & 0 \\
MANCHEGO & 19.240 & 10 \\
PP & & \\
\hline
\end{tabular}




\section{PROVINCIA:}

J. ELECTORAL DE ZONA: ALICANTE MUNICIPIO:

\section{ALICANTE}

\section{ALICANTE}

\begin{tabular}{lr}
\hline N. ${ }^{\circ}$ electores: & 201.391 \\
N. ${ }^{\circ}$ votantes: & 113.010 \\
N. ${ }^{\circ}$ votos de candidatura: & 111.407 \\
N. ${ }^{\circ}$ votos en blanco: & 1.171 \\
N. ${ }^{\circ}$ votos válidos: & 112.578 \\
N. ${ }^{\circ}$ votos nulos: & 432 \\
\hline
\end{tabular}

\begin{tabular}{|l|r|r|}
\hline CANDIDATURAS & $\begin{array}{c}\text { VotOS } \\
\text { OBTENIDOS }\end{array}$ & $\begin{array}{c}\text { N. }{ }^{\circ} \text { coNCEJ. } \\
\text { ELECTOS }\end{array}$ \\
\hline CDS & 4.006 & 0 \\
PP & 40.021 & 12 \\
UNIÓ VALENCIÁ & 665 & 0 \\
UNITAT DEL POBLE VALENCIA & 714 & 0 \\
UNION DEMOCRATICA & 1.600 & 0 \\
ALICANTINA & & \\
ESQUERRA UNIDA DEL PAIS & 9.545 & 2 \\
VALENCIÁ & 1.679 & 0 \\
LOS VERDES DE ALICANTE & 6.623 & 1 \\
UNION VERDE & 43.079 & 12 \\
PARTIDO SOLIDARIDAD CIVICA & 261 & 0 \\
PSOE & 1.019 & 0 \\
PARTIDO NACIONAL & & \\
ALICANTINOS INDEPEND. & 252 & 0 \\
PARTIDO CANTONALISTA DEL & 1.943 & 0 \\
PAÍS ALICANTINO & & \\
FALANGE ESPAÑOLA DE LAS & & \\
JONS & & \\
LOS VERDES & & \\
\hline
\end{tabular}


PROVINCIA:

ALMERÍA

J. ELECTORAL DE ZONA: ALMERIA

MUNICIPIO:

ALMERIA
$N{ }^{\circ}$ electores:
116.779
N. votantes:
61.504
N. ${ }^{\circ}$ votos de candidatura:
60.914
N. ${ }^{\circ}$ votos en blanco:
423
$\mathrm{N} .^{\circ}$ votos válidos:
61.337
N. ${ }^{\circ}$ votos nulos:
167

\begin{tabular}{|l|r|r|}
\hline CANDIDATURAS & $\begin{array}{c}\text { VotOS } \\
\text { OBTENIDOS }\end{array}$ & $\begin{array}{c}\text { N. }{ }^{\circ} \text { CONCEJ. } \\
\text { ELECTOS }\end{array}$ \\
\hline PIAP & 514 & 0 \\
UNAL & 1.664 & 0 \\
DS & 622 & 0 \\
PP & 20.965 & 10 \\
VERDES & 866 & 0 \\
IU-CA & 5.798 & 3 \\
PARTIDO ANDALUCISTA & 3.922 & 2 \\
CDS & 1.573 & 0 \\
PSOE & 24.796 & 12 \\
VLEH & 194 & 0 \\
\hline
\end{tabular}


PROVINCIA:

ASTURIAS

J. ELECTORAL DE ZONA: OVIEDO

MUNICIPIO:

OVIEDO

N. ${ }^{\circ}$ electores:

158.425

N. ${ }^{\circ}$ votantes:

90.165

$N .^{\circ}$ votos de candidatura:

88.245

$\mathrm{N} .^{\circ}$ votos en blanco:

1.475

N. ${ }^{\circ}$ votos válidos:

89.720

$\mathrm{N} .^{\circ}$ votos nulos:

445

\begin{tabular}{|c|c|c|}
\hline CandidatuRas & $\begin{array}{c}\text { VOTOS } \\
\text { OBTENIDOS }\end{array}$ & $\begin{array}{l}\text { N. }{ }^{\circ} \text { CONCEJ } \\
\text { ELECTOS }\end{array}$ \\
\hline PSOE & 30.634 & 10 \\
\hline CONVERGENCIA ASTURIANA & 624 & 0 \\
\hline CDS & 5.803 & 2 \\
\hline ANDECHA ASTUR & 207 & 0 \\
\hline LOS VERDES & 1.300 & 0 \\
\hline IU & 8.635 & 2 \\
\hline CA PAS-UNA & 1.773 & 0 \\
\hline $\begin{array}{l}\text { CONCEYU INDEPENDIENTE } \\
\text { D ASTURIES }\end{array}$ & 262 & 0 \\
\hline PP & 39.007 & 13 \\
\hline
\end{tabular}


PROVINCIA:

ÁVILA

J. ELECTORAL DE ZONA: ÁVILA

MUNICIPIO:

ÁVILA

N. ${ }^{\circ}$ electores:

34.742

N. votantes:

21.096

$\mathrm{N} .{ }^{\circ}$ votos de candidatura:

20.505

$N^{\circ}$ votos en blanco:

403

N. ${ }^{\circ}$ votos válidos:

20.919

$\mathrm{N} .^{\circ}$ votos nulos:

177

\begin{tabular}{|l|c|c|}
\hline CANDIDATURAS & $\begin{array}{c}\text { VoTOS } \\
\text { OBTENIDOS }\end{array}$ & $\begin{array}{c}\text { N. }{ }^{\circ} \text { CONCEJ. } \\
\text { ELECTOS }\end{array}$ \\
\hline PP & 9.763 & 11 \\
CDS & 4.358 & 5 \\
PSOE & 3.823 & 4 \\
IU & 1.577 & 1 \\
LOS VERDES & 995 & 0 \\
\hline
\end{tabular}


PROVINCIA:

J. ELECTORAL DE ZONA: BADAJOZ

MUNICIPIO:

\section{BADAJOZ}

BADAJOZ

\begin{tabular}{|c|c|c|c|}
\hline & N. ${ }^{\circ}$ electores: & \multicolumn{2}{|c|}{92.013} \\
\hline & $\mathrm{N}^{\circ}$ votantes: & \multicolumn{2}{|c|}{53.032} \\
\hline & N. ${ }^{\circ}$ votos de candidatura: & \multicolumn{2}{|c|}{25.040} \\
\hline & N. ${ }^{\circ}$ votos en blanco: & \multicolumn{2}{|c|}{613} \\
\hline & N. ${ }^{\circ}$ votos válidos: & \multicolumn{2}{|c|}{52.653} \\
\hline . & N. ${ }^{\circ}$ votos nulos: & \multicolumn{2}{|c|}{379} \\
\hline CANDIDATURAS & & $\begin{array}{c}\text { VOTOS } \\
\text { OBTENIDOS }\end{array}$ & $\begin{array}{l}\text { N. }{ }^{\circ} \text { CONCEJ. } \\
\text { ELECTOS }\end{array}$ \\
\hline PSOE & & 26.874 & 15 \\
\hline PP & & 17.723 & 10 \\
\hline IU & & 3.927 & 2 \\
\hline
\end{tabular}


PROVINCIA:

BALEARES

J. ELECTORAL DE ZONA: PALMA

MUNICIPIO:

PALMA
N. ${ }^{\circ}$ electores:
244.209
N. ${ }^{\circ}$ votantes:
125.083
N. ${ }^{\circ}$ votos de candidatura:
122.951
N. votos en blanco:
1.350
N. ${ }^{\circ}$ votos válidos:
124.301
N. ${ }^{\circ}$ votos nulos:
782

\begin{tabular}{|c|c|c|}
\hline Candidaturas & $\begin{array}{l}\text { VOTOS } \\
\text { OBTENIDOS }\end{array}$ & $\begin{array}{l}\text { N. } .^{\circ} \text { CONCEJ } \\
\text { ELECTOS }\end{array}$ \\
\hline PP-UNIÓN MALLORQUINA & 59.420 & 17 \\
\hline $\begin{array}{l}\text { COALICION ALIANZA POR LA } \\
\text { REPÚBLICA }\end{array}$ & 477 & 0 \\
\hline PSOE & 37.936 & 10 \\
\hline PARTIDO RADICAL BALEAR & 202 & 0 \\
\hline CONVERGENCIA BALEAR & 995 & 0 \\
\hline $\begin{array}{l}\text { IU-ESQUERRA UNIDA DE } \\
\text { MALLORCA }\end{array}$ & 5.250 & 0 \\
\hline $\begin{array}{l}\text { PSM-NACIONALISTAS DE } \\
\text { MALLORCA }\end{array}$ & 1.952 & 2 \\
\hline ELS VERDS & 4.487 & 0 \\
\hline $\begin{array}{l}\text { UNION INDEPENDIENTE DE } \\
\text { MALLORCA }\end{array}$ & 3.589 & 0 \\
\hline CDS & 2.933 & 0 \\
\hline $\begin{array}{l}\text { PARTIDO FALANGE ESPAÑOLA } \\
\text { DE LAS JONS }\end{array}$ & 310 & 0 \\
\hline
\end{tabular}


PROVINCIA:

BARCELONA

J. ELECTORAL DE ZONA: BARCELONA

MUNICIPIO:

BARCELONA
N. ${ }^{\circ}$ electores:
1.380 .587
N. ${ }^{\circ}$ votantes:
766.520
$\mathrm{N} .{ }^{\circ}$ votos de candidatura:
757.364
N. ${ }^{\circ}$ votos en blanco:
6.979
$\mathrm{N} .{ }^{\circ}$ votos válidos:
764.343
N. ${ }^{\circ}$ votos nulos:
2.177

\begin{tabular}{|c|c|c|}
\hline Candidaturas & $\begin{array}{l}\text { VOTOS } \\
\text { OBTENIDOS }\end{array}$ & $\begin{array}{l}\text { N. }{ }^{\circ} \text { CONCEJ. } \\
\text { ELECTOS }\end{array}$ \\
\hline $\begin{array}{l}\text { PARTIT DELS SOCIALISTES DE } \\
\text { CATALUNYA }\end{array}$ & 328.282 & 20 \\
\hline INICIATIVA PER CATALUNYA & 49.034 & 3 \\
\hline $\begin{array}{l}\text { PARTIR DELS COMUNISTES DE } \\
\text { CATALUNYA }\end{array}$ & 3.168 & 0 \\
\hline $\begin{array}{l}\text { ESQUERRA REPUBLICANA DE } \\
\text { CATALUNYA }\end{array}$ & 19.629 & 0 \\
\hline CONVERGENCIA I UNIÓ & 260.344 & 16 \\
\hline UNIDAD CENTRISTA CATALANA & 198 & 0 \\
\hline $\operatorname{CDS}$ & 6.454 & 0 \\
\hline $\begin{array}{l}\text { ALTERNATIVA PARA } \\
\text { BARCELONA }\end{array}$ & 428 & 0 \\
\hline $\begin{array}{l}\text { PARTIDO SOCIALISTA DE LOS } \\
\text { TRABAJADORES }\end{array}$ & 1.308 & 0 \\
\hline BARCELONA VERDA & 5.749 & 0 \\
\hline PLATAFORMA DE ESQUERRES & 210 & $\mathbf{0}$ \\
\hline $\begin{array}{l}\text { CENTRE RADICAL DE } \\
\text { CATALUNYA }\end{array}$ & 294 & 0 \\
\hline $\begin{array}{l}\text { PARTIT OBRER } \\
\text { REVOLUCIONARI DE ESPANYA }\end{array}$ & 74.804 & 4 \\
\hline PP & 74.804 & 4 \\
\hline $\begin{array}{l}\text { LOS VERDES LISTA } \\
\text { ECOLOGISTA HUMANISTA .. }\end{array}$ & 6.120 & 0 \\
\hline ALIANZA PER LA REPÚBLICA & 296 & 0 \\
\hline $\begin{array}{l}\text { PARTIDO ACCION SOCIAL } \\
\text { EVANGELISTA }\end{array}$ & 514 & 0 \\
\hline
\end{tabular}


PROVINCIA:

J. ELECTORAL DE ZONA: BURGOS

MUNICIPIO:

\section{BURGOS}

\section{BURGOS}
N. ${ }^{\circ}$ electores:
125.288
N. ${ }^{\circ}$ votantes:
79.372
$\mathrm{N}^{\circ}{ }^{\circ}$ votos de candidatura:
77.542
$N .^{\circ}$ votos en blanco:
1.450
N. ${ }^{\circ}$ votos válidos:
78.992
N. ${ }^{\circ}$ votos nulos:
638

\begin{tabular}{|l|c|c|}
\hline CANDIDATURAS & $\begin{array}{c}\text { VoTOS } \\
\text { OBTENIDOS }\end{array}$ & $\begin{array}{c}\text { N. }{ }^{\circ} \text { CONCEJ. } \\
\text { ELECTOS }\end{array}$ \\
\hline PP & 33.438 & 14 \\
PSOE & 25.541 & 11 \\
IU & 5.966 & 2 \\
CDS & 3.397 & 0 \\
APB & 3.289 & 0 \\
UBI & 3.193 & 0 \\
PREC & 1.167 & 0 \\
LOS VERDES & 1.066 & 0 \\
TC & 485 & 0 \\
\hline
\end{tabular}




\section{PROVINCIA:}

\section{CÁCERES}

\section{J. ELECTORAL DE ZONA: CACERES}

MUNICIPIO:

CACERES

\begin{tabular}{lr}
\hline N. ${ }^{\circ}$ electores: & 55.178 \\
N. ${ }^{\circ}$ votantes: & 33.738 \\
N. ${ }^{\circ}$ votos de candidatura: & 33.124 \\
N. ${ }^{\circ}$ votos en blanco: & 394 \\
N. ${ }^{\circ}$ votos válidos: & 33.518 \\
N. ${ }^{\circ}$ votos nulos: & $\cdot 220$ \\
\hline
\end{tabular}

\begin{tabular}{|l|c|c|}
\hline CANDIDATURAS & $\begin{array}{c}\text { VotOS } \\
\text { OBTENIDOS }\end{array}$ & $\begin{array}{c}\text { N. }^{\circ} \text { CONCEJ. } \\
\text { ELECTOS }\end{array}$ \\
\hline PP & 13.218 & 12 \\
CDS & 1.505 & 0 \\
C. INDEPENDIENTE CACERENA & 1.335 & 0 \\
PSOE & 12.928 & 12 \\
EU & 1.688 & 1 \\
PREX & 791 & 0 \\
IU & 1.659 & 0 \\
\hline
\end{tabular}


PROVINCIA:

CÁDIZ

J. ELECTORAL DE ZONA: CÁDIZ

MUNICIPIO:

CÁDIZ

N. ${ }^{\circ}$ electores:

116.824

$\mathrm{N} .^{\circ}$ votantes:

52.992

$N .^{\circ}$ votos de candidatura:

51.802

N. votos en blanco:

783

N. ${ }^{\circ}$ votos válidos:

52.585

N. ${ }^{\circ}$ votos nulos:

407

\begin{tabular}{|l|c|c|}
\hline CANDIDATURAS & $\begin{array}{c}\text { VotOS } \\
\text { OBTENIDOS }\end{array}$ & $\begin{array}{c}\text { N. }{ }^{\circ} \text { CONCEJ. } \\
\text { ELECTOS }\end{array}$ \\
\hline CDS & 1.390 & 0 \\
PSOE & 24.449 & 14 \\
PP & 13.322 & 7 \\
IU-CA & 6.450 & 3 \\
PARTIDO ANDALUCISTA & 5.701 & 3 \\
UNIDAD POPULAR ANDALUZA & 266 & 0 \\
FALANGE ESPAÑOLA DE LAS JONS & 224 & 0 \\
\hline
\end{tabular}


PROVINCIA:

J. ELECTORAL DE ZONA: SANTANDER

MUNICIPIO:
CANTABRIA

SANTANDER
N. ${ }^{\circ}$ electores:
147.212
N. ${ }^{\circ}$ votantes:
90.572
N. ${ }^{\circ}$ votos de candidatura:
87.329
$\mathrm{N} .^{\circ}$ votos en blanco:
2.352
N. ${ }^{\circ}$ votos válidos:
89.681
N. ${ }^{\circ}$ votos nulos:
891

\begin{tabular}{|c|c|c|}
\hline CANDIDATURAS & $\begin{array}{c}\text { VOTOS } \\
\text { OBTENIDOS }\end{array}$ & $\begin{array}{l}\text { N. }^{\circ} \text { CONCEJ. } \\
\text { ELECTOS }\end{array}$ \\
\hline PSOE & 28.808 & 10 \\
\hline IU & 4.519 & 1 \\
\hline $\begin{array}{l}\text { PARTIDO COMUNISTA DE LOS } \\
\text { PUEBLOS DE ESPAÑA }\end{array}$ & 348 & 0 \\
\hline CDS & 1.897 & 0 \\
\hline $\begin{array}{l}\text { PARTIDO REGIONALISTA DE } \\
\text { CANTABRIA }\end{array}$ & 4.443 & 0 \\
\hline PP & 17.141 & 6 \\
\hline $\begin{array}{l}\text { PARTIDO NACIONALISTA DE } \\
\text { CANTABRIA }\end{array}$ & 293 & 0 \\
\hline $\begin{array}{l}\text { AGRUPACIÓN VECINAL DE } \\
\text { CANTABRIA }\end{array}$ & 1.688 & 0 \\
\hline $\begin{array}{l}\text { LOS VERDES LISTA } \\
\text { ECOLÓGICA-HUMANISTA }\end{array}$ & 720 & 0 \\
\hline $\begin{array}{l}\text { UNION PARA EL PROGRESO DE } \\
\text { CANTABRIA }\end{array}$ & 27.472 & 10 \\
\hline
\end{tabular}




\section{PROVINCIA: $\quad$ CASTELLON}

\section{J. ELECTORAL DE ZONA: CASTELLONN}

MUNICIPIO:
N. ${ }^{\circ}$ electores:
101.363
N. ${ }^{\circ}$ votantes:
61.151
$\mathrm{N} .{ }^{\circ}$ votos de candidatura:
0
N. ${ }^{\circ}$ votos en blanco:
765
N. ${ }^{\circ}$ votos válidos:
0
N. ${ }^{\circ}$ votos nulos:
332

\begin{tabular}{|l|c|c|}
\hline CANDIDATURAS & $\begin{array}{c}\text { VotOS } \\
\text { OBTENIDOS }\end{array}$ & $\begin{array}{c}\text { N. }{ }^{\circ} \text { CONCEJ. } \\
\text { ELECTOS }\end{array}$ \\
\hline PSOE & 21.814 & 13 \\
ESQUERRA UNIDA PAIS VALENCIA & 2.659 & 0 \\
DEMOCRACIA SOCIALISTA & 1.666 & 0 \\
PP & 23.325 & 14 \\
UNITAT DEL POBLE VALENCIA & 2.995 & 0 \\
LOS VERDES & 1.719 & 0 \\
GRAU UNIT & 1.757 & 0 \\
CDS & 2.137 & 0 \\
\hline
\end{tabular}


PROVINCIA:

CEUTA

J. ELECTORAL DE ZONA: CEUTA

MUNICIPIO:

CEUTA
N. ${ }^{\circ}$ electores:
43.135
N..$^{\circ}$ votantes:
25.163
N. ${ }^{\circ}$ votos de candidatura:
25.166
N. ${ }^{\circ}$ votos en blanco:
119
$N .^{\circ}$ votos válidos:
25.285
N. ${ }^{\circ}$ votos nulos:
107

\begin{tabular}{|l|c|c|}
\hline CANDIDATURAS & $\begin{array}{c}\text { Votos } \\
\text { OBTENIDOS }\end{array}$ & $\begin{array}{c}\text { N. }{ }^{\circ} \text { CONCEJ. } \\
\text { ELECTOS }\end{array}$ \\
\hline PP & 5.812 & 6 \\
PROGRESO Y.FUTURO DE CEUTA & 9.420 & 11 \\
PSOE & 3.004 & 3 \\
PARTIDO CEUTA UNIDA & 2.501 & 2 \\
PARTIDO SOCIALISTA DEL PUEBLO & 2.065 & 2 \\
DE CEUTA & 202 & 0 \\
PST & 239 & 0 \\
PARTIDO INICIATIVA POR CEUTA & 417 & 0 \\
LOS VERDES & 1.273 & 1 \\
CDS & 233 & 0 \\
PARTIDO HUMANISTA & & $\vdots$ \\
\hline
\end{tabular}


PROVINCIA:

CIUDAD REAL

J. ELECTORAL DE ZONA: CIUDAD REAL MUNICIPIO:
N..$^{\circ}$ electores:
42.127
N. ${ }^{\circ}$ votantes:
23.808
N. ${ }^{\circ}$ votos de candidatura:
23.130
$\mathrm{N} .{ }^{\circ}$ votos en blanco:
394
N. ${ }^{\circ}$ votos válidos:
23.524
N. . votos nulos:
284

\begin{tabular}{|l|c|c|}
\hline CANDIDATURAS & $\begin{array}{c}\text { VOTOS } \\
\text { OBTENIDOS }\end{array}$ & $\begin{array}{c}\text { N. }{ }^{\circ} \text { CONCEJ. } \\
\text { ELECTOS }\end{array}$ \\
\hline $\begin{array}{l}\text { PARTIDO UNITARIO } \\
\text { REGIONALISTA }\end{array}$ & 511 & 0 \\
IU & 1.795 & 2 \\
PP & 9.260 & 10 \\
PSOE & 9.855 & 11 \\
CDS & 1.709 & 2 \\
\hline
\end{tabular}


PROVINCIA:

CORDOBA

J. ELECTORAL DE ZONA: CÓRDOBA

MUNICIPIO:

CORDOBA
N. ${ }^{\circ}$ electores:
226.318
N. ${ }^{\circ}$ votantes:
121.219
N. ${ }^{\circ}$ votos de candidatura:
119.483
$\mathrm{N} .^{\circ}$ votos en blanco:
1.007
N. ${ }^{\circ}$ votos válidos:
120.490
N. ${ }^{\circ}$ votos nulos:
518

\begin{tabular}{|l|c|c|}
\hline CANDIDATURAS & $\begin{array}{c}\text { VOTOS } \\
\text { OBTENIDOS }\end{array}$ & $\begin{array}{c}\text { N. }{ }^{\circ} \text { CONCEJ. } \\
\text { ELECTOS }\end{array}$ \\
\hline PSOE-A & 35.771 & 9 \\
PA & 5.966 & 0 \\
CDS & 1.642 & 0 \\
PP & 28.113 & 7 \\
IU-CA & 48.051 & 13 \\
\hline
\end{tabular}


PROVINCIA:

J. ELECTORAL DE ZONA: CUENCA MUNICIPIO:

\section{CUENCA}

CUENCA
N. ${ }^{\circ}$ electores:
33.150
N. ${ }^{\circ}$ votantes:
22.226
N. ${ }^{\circ}$ votos de candidatura:
21.778
$\mathrm{N} .^{\circ}$ votos en blanco:
298
$\mathrm{N}{ }^{\circ}$ votos válidos:
22.076
N. ${ }^{\circ}$ votos nulos:
150

\begin{tabular}{|l|c|c|}
\hline CANDIDATURAS & $\begin{array}{c}\text { VOTOS } \\
\text { OBTENIDOS }\end{array}$ & $\begin{array}{c}\text { N. }{ }^{\circ} \text { CONCEJ. } \\
\text { ELECTOS }\end{array}$ \\
\hline PSOE & 10.649 & 11 \\
IU & 891 & 0 \\
PP & 9.373 & 10 \\
CDS & 530 & 0 \\
TIERRA COMUNERA & 335 & 0 \\
\hline
\end{tabular}


PROVINCIA:

J. ELECTORAL DE ZONA: GERONA

MUNICIPIO:

\section{GERONA}

\section{GERONA}
N. ${ }^{\circ}$ electores:
52.703
N. ${ }^{\circ}$ votantes:
30.540
N. ${ }^{\circ}$ votos de candidatura:
30.052
N. ${ }^{\circ}$ votos en blanco:
333
N. ${ }^{\circ}$ votos válidos:
30.385
N. ${ }^{\circ}$ votos nulos:
155

\begin{tabular}{|l|c|c|}
\hline CANDIDATURAS & $\begin{array}{c}\text { VOTOS } \\
\text { OBTENIDOS }\end{array}$ & $\begin{array}{c}\text { N. }{ }^{\circ} \text { CONCEJ. } \\
\text { ELECTOS }\end{array}$ \\
\hline PSC-PSOE & 14.451 & 13 \\
CIU & 9.361 & 9 \\
PP & 2.103 & 2 \\
ERC & 1.729 & 1 \\
IU & 1.250 & 0 \\
\hline
\end{tabular}


PROVINCIA:

GRANADA

J. ELECTORAL DE ZONA: GRANADA

MUNICIPIO:

GRANADA

N. electores:

201.703

N. ${ }^{\circ}$ votantes:

110.550

N. ${ }^{\circ}$ votos de candidatura:

109.227

$\mathrm{N} .^{\circ}$ votos en blanco:

877

$N{ }^{\circ}$ votos válidos:

110.104

$N:{ }^{\circ}$ votos nulos:

426

\begin{tabular}{|l|c|c|}
\hline CANDIDATURAS & $\begin{array}{c}\text { VotOS } \\
\text { OBTENIDOS }\end{array}$ & $\begin{array}{c}\text { N. }{ }^{\circ} \text { CONCEJ. } \\
\text { ELECTOS }\end{array}$ \\
\hline UNIDAD GRANADINA & 2.185 & 0 \\
PSOE-A & 42.390 & 12 \\
IU-CA & 9.811 & 2 \\
PP & 45.256 & 13 \\
CDS & 1.924 & 0 \\
PA & 4.686 & 0 \\
UNIDAD POPULAR DE & 126 & 0 \\
ANDALUCIA & 144 & 0 \\
FALANGE ESPANOOLA DE LAS & 2.274 & 0 \\
JONS & & 0 \\
COS VERDES DE ANDALUCIA & 431 & \\
HUMANISTA & & \\
\hline
\end{tabular}


PROVINCIA:

\section{GUADALAJARA}

J. ELECTORAL DE ZONA: GUADALAJARA

MUNICIPIO: $\quad$ GUADALAJARA

\begin{tabular}{|c|c|c|c|}
\hline & N. ${ }^{\circ}$ electores: & \multicolumn{2}{|c|}{47.542} \\
\hline & N..$^{\circ}$ votantes: & \multicolumn{2}{|c|}{31.075} \\
\hline & $\mathrm{N}^{\circ}{ }^{\circ}$ votos de candidatura: & \multicolumn{2}{|c|}{30.023} \\
\hline & N. ${ }^{\circ}$ votos en blanco: & \multicolumn{2}{|c|}{642} \\
\hline & N. ${ }^{\circ}$ votos válidos: & \multicolumn{2}{|c|}{30.665} \\
\hline & N. ${ }^{\circ}$ votos nulos: & \multicolumn{2}{|c|}{410} \\
\hline \multicolumn{4}{|c|}{\lrcorner} \\
\hline CANDIDATURAS & & $\begin{array}{c}\text { VOTOS } \\
\text { OBTENIDOS }\end{array}$ & $\begin{array}{l}\text { N. }{ }^{\circ} \text { CONCEJ. } \\
\text { ELECTOS }\end{array}$ \\
\hline PP & & 13.404 & 12 \\
\hline PSOE & . & 11.503 & 10 \\
\hline IU & & 3.388 & 3 \\
\hline
\end{tabular}


PROVINCIA:

GUUIPÚzCOA

J. ELECTORAL DE ZONA: DONOSTIA-SAN SEBASTIAN MUNICIPIO: DONOSTIA-SAN SEBASTIAN
$\dot{N} .^{\circ}$ electores:
143.094
N. ${ }^{\circ}$ votantes:
80.274
N. ${ }^{\circ}$ votos de candidatura:
79.082
N. ${ }^{\circ}$ votos en blanco:
792
N. ${ }^{\circ}$ votos válidos:
79.874
N. ${ }^{\circ}$ votos nulos:
400

\begin{tabular}{|l|c|c|}
\hline CANDIDATURAS & $\begin{array}{c}\text { VoTOS } \\
\text { OBTENIDOS }\end{array}$ & $\begin{array}{c}\text { N. }^{\circ} \text { CONCEJ. } \\
\text { ELECTOS }\end{array}$ \\
\hline PSE-PSOE & 13.526 & 5 \\
EAJ-PNV & 12.170 & 4 \\
EE & 6.922 & 2 \\
HB & 13.791 & 5 \\
PP & 13.036 & 5 \\
PLATAFORMA IZQUIERDA & 82 & 0 \\
CDS & 300 & 0 \\
EUSKALHERRICO BERDEAK & 587 & 0 \\
IU & 809 & 0 \\
EA & 17.859 & 6 \\
\hline
\end{tabular}


PROVINCIA:

\section{HUELVA}

J. ELECTORAL DE ZONA: HUELVA

MUNICIPIO:

HUELVA

\begin{tabular}{lc}
\hline N. ${ }^{\circ}$ electores: & 101.795 \\
N. ${ }^{\circ}$ votantes: & 45.444 \\
$\mathrm{~N}^{\circ}$ votos de candidatura: & 44.965 \\
$\mathrm{~N}^{\circ}$ votos en blanco: & 310 \\
$\mathrm{~N}^{\circ}$ votos válidos: & 45.375 \\
$\mathrm{~N} .{ }^{\circ}$ votos nulos: & 169 \\
\hline
\end{tabular}

\begin{tabular}{|l|c|c|}
\hline CANDIDATURAS & $\begin{array}{c}\text { VoTOS } \\
\text { OBTENIDOS }\end{array}$ & $\begin{array}{c}\text { N. }{ }^{\circ} \text { CONCEJ. } \\
\text { ELECTOS }\end{array}$ \\
\hline PSOE-A & 23.326 & 16 \\
IU-CA & 4.358 & 2 \\
PARTIDO ANDALUCISTA & 3.221 & 2 \\
PP & 11.201 & 7 \\
\hline
\end{tabular}


PROVINCIA:

HUESCA

J. ELECTORAL DE ZONA: HUESCA

MUNICIPIO:

HUESCA

\begin{tabular}{lr}
\hline N. ${ }^{\circ}$ electores: & 33.456 \\
N. ${ }^{\circ}$ votantes: & 20.152 \\
N. ${ }^{\circ}$ votos de candidatura: & 19.743 \\
N. ${ }^{\circ}$ votos en blanco: & 287 \\
N..$^{\circ}$ votos válidos: & 20.030 \\
N..$^{\circ}$ votos nulos: & 122 \\
\hline
\end{tabular}

\begin{tabular}{|l|c|c|}
\hline CANDIDATURAS & $\begin{array}{c}\text { VOIOS } \\
\text { OBTENIDOS }\end{array}$ & $\begin{array}{c}\text { N. }{ }^{\circ} \text { CONCEJ. } \\
\text { ELECTOS }\end{array}$ \\
\hline PSOE & 6.664 & 8 \\
PP & 4.730 & 5 \\
PAR & 4.423 & 5 \\
CONVERGENCIA ALT. & 1.835 & 2 \\
ARAGÓN-IU & 1.347 & 1 \\
CDS & 735 & \\
CHUNTA ARAGONESISTA & & \\
\hline
\end{tabular}




\section{PROVINCIA:}

\section{JAÉN}

J. ELECTORAL DE ZONA: JAÉN

MUNICIPIO: JAÉN
N. ${ }^{\circ}$ electores:
77.750
N..$^{\circ}$ votantes:
47.176
N. ${ }^{\circ}$ votos de candidatura:
46.720
N. ${ }^{\circ}$ votos en blanco:
270
N. ${ }^{\circ}$ votos válidos:
46.990
N. ${ }^{\circ}$ votos nulos:
186

\begin{tabular}{|l|c|c|}
\hline CANDIDATURAS & $\begin{array}{c}\text { VotOS } \\
\text { OBTENIDOS }\end{array}$ & $\begin{array}{c}\text { N. }{ }^{\circ} \text { CONCEJ. } \\
\text { ELECTOS }\end{array}$ \\
\hline PA & 2.716 & 1 \\
OCTUBRE SOCIALISTA & 237 & 0 \\
NACIÓN ANDALUZA & 103 & 0 \\
DEMOCRACIA SOCIALISTA & 1.431 & 0 \\
PP & 19.261 & 12 \\
PSOE-A & 18.803 & 12 \\
IU-CA & 3.299 & 2 \\
FALANGE ESPAÑOLA DE LAS JONS & 72 & 0 \\
CDS & 798 & 0 \\
\hline
\end{tabular}


PROVINCIA:

\section{LEON}

J. ELECTORAL DE ZONA: LEON

MUNICIPIO:

LEON

N. ${ }^{\circ}$ electores:

106.042

N. ${ }^{\circ}$ votantes:

62.043

$\mathrm{N} .{ }^{\circ}$ votos de candidatura:

59.796

N. ${ }^{\circ}$ votos en blanco:

1.631

N. ${ }^{\circ}$ votos válidos:

61.427

$N .^{\circ}$ votos nulos:

616

\begin{tabular}{|l|c|c|}
\hline CANDIDATURAS & $\begin{array}{c}\text { VOTOS } \\
\text { OBTENIDOS }\end{array}$ & $\begin{array}{c}\text { N. } .^{\circ} \text { CONCEJ. } \\
\text { ELECTOS }\end{array}$ \\
\hline $\begin{array}{l}\text { MOVIMIENTO FALANGISTA DE } \\
\text { ESPAÑA }\end{array}$ & 228 & 0 \\
PP & 24.944 & 13 \\
PSOE & 18.857 & 10 \\
CDS & 2.799 & 0 \\
UNION DEL PUEBLO LEONES & 6.236 & 3 \\
LOS VERDES & 1.024 & 0 \\
UPI & 2.540 & 0 \\
IU & 3.168 & 1 \\
\hline
\end{tabular}


PROVINCIA:

\section{LA CORUNAA}

J. ELECTORAL DE ZONA: LA CORUÑA MUNICIPIO: LA CORUÑA

N. ${ }^{\circ}$ electores:

N..$^{\circ}$ votantes:

N. ${ }^{\circ}$ votos de candidatura:

$\mathrm{N}^{\circ}$ votos en blanco:

N. ${ }^{\circ}$ votos válidos:

N. ${ }^{\circ}$ votos nulos:
198.781

112.249

111.027

1.089

112.116

300

\begin{tabular}{|l|c|c|}
\hline CANDIDATURAS & $\begin{array}{c}\text { VoTOS } \\
\text { OBTENIDOS }\end{array}$ & $\begin{array}{c}\text { N. }^{\circ} \text { CONCEJ. } \\
\text { ELECTOS }\end{array}$ \\
\hline PS DE G PSOE & 66.445 & 18 \\
PP & 32.353 & 9 \\
BNG & 5.389 & \\
PSG EG & 2.288 & \\
EU & 2.121 & \\
CDS & 1.368 & \\
LVLEH & 570 & \\
PSY & 344 & 149 \\
PG &
\end{tabular}


PROVINCIA:

LAS PALMAS

\section{J. ELECTORAL DE ZONA: LAS PALMAS DE GRAN CANARIA} MUNICIPIO:
N. ${ }^{\circ}$ electores:
275.298
N. votantes:
147.260
N. ${ }^{\circ}$ votos de candidatura:
146.027
N. ${ }^{\circ}$ votos en blanco:
1.579
$\mathrm{N} .^{\circ}$ votos válidos:
147.606
N. ${ }^{\circ}$ votos nulos:
1.233

\begin{tabular}{|l|c|c|}
\hline CANDIDATURAS & $\begin{array}{c}\text { VOTOS } \\
\text { OBTENIDOS }\end{array}$ & $\begin{array}{c}\text { N. }^{\circ} \text { CONCEJ. } \\
\text { ELECTOS }\end{array}$ \\
\hline PSOE & 42.179 & 10 \\
PP & 31.815 & 7 \\
IGC & 3.808 & 0 \\
PLATAFORMA DE IZQUIERDAS & 845 & 0 \\
LOS VERDES LISTA & 761 & 0 \\
ECOLOGISTA-HUMANISTA & 527 & 0 \\
AGRUPACION INSULAR DE GRAN & & 0 \\
CANARIA & 3.555 & 0 \\
PARTIDO NACIONALISTA & 24.437 & 5 \\
CANARIO & 33.359 & 7 \\
ICAN & 1.535 & 0 \\
CDS & 472 & 0 \\
LOS VERDES & 282 & 0 \\
PARTIDO TAGOROR & & 0 \\
PARTIDO LA GENTE & & 0 \\
COAL. ELECTORAL CANARIAS POR & & 0 \\
LA INDEPENDENCIA & & 0 \\
\hline
\end{tabular}




\section{PROVINCIA:}

\section{LA RIOJA}

J. ELECTORAL DE ZONA: LOGROÑO

MUNICIPIO:

\section{LOGROÑO}
N. ${ }^{\circ}$ electores:
93.504
N..$^{\circ}$ votantes:
56.624
N. ${ }^{\circ}$ votos de candidatura:
55.096
N. ${ }^{\circ}$ votos en blanco:
1.075
N. ${ }^{\circ}$ votos válidos:
56.171
N. ${ }^{\circ}$ votos nulos:
453

\begin{tabular}{|l|c|c|}
\hline CandidATURAS & $\begin{array}{c}\text { VotOS } \\
\text { OBTENIDOS }\end{array}$ & $\begin{array}{c}\text { N. }{ }^{\circ} \text { conCEJ. } \\
\text { ELECTOS }\end{array}$ \\
\hline PP & 23.406 & 13 \\
PSOE & 22.989 & 12 \\
CDS & 3.479 & 1 \\
IU & 3.355 & 1 \\
\hline
\end{tabular}


PROVINCIA:

\section{LERIDA}

J. ELECTORAL DE ZONA: LÉRIDA

MUNICIPIO:

LÉRIDA

N. ${ }^{\circ}$ electores:

87.362

N. ${ }^{\circ}$ votantes:

49.135

$N .^{\circ}$ votos de candidatura:

48.012

N. ${ }^{\circ}$ votos en blanco:

649

N. ${ }^{\circ}$ votos válidos:

48.661

N. ${ }^{\circ}$ votos nulos:

374

\begin{tabular}{|l|c|c|}
\hline CANDIDATURAS & $\begin{array}{c}\text { VoTOS } \\
\text { OBTENIDOS }\end{array}$ & $\begin{array}{c}\text { N. }^{\circ} \text { CONCEJ. } \\
\text { ELECTOS }\end{array}$ \\
\hline GIF & 2.349 & 17 \\
PSC-PSOE & 24.029 & \\
IC-AV & 2.400 & \\
PCC & 333 & \\
CDS & 781 & \\
ERC & 1.677 & 8 \\
PP & 3.911 & \\
AIPN & 79 & \\
CIU & 12.453 & \\
\hline
\end{tabular}




\section{PROVINCIA:}

J. ELECTORAL DE ZONA: LUGO

MUNICIPIO:

\section{LUGO}

LUGO
N. ${ }^{\circ}$ electores:
67.042
N. ${ }^{\circ}$ votantes:
39.343
N. ${ }^{\circ}$ votos de candidatura:
38.630
N. ${ }^{\circ}$ votos en blanco:
507
N. ${ }^{\circ}$ votos válidos:
39.137
N. ${ }^{\circ}$ votos nulos:
206

\begin{tabular}{|l|c|c|}
\hline CANDIDATURAS & $\begin{array}{c}\text { VoTOS } \\
\text { OBTENIDOS }\end{array}$ & $\begin{array}{c}\text { N. }{ }^{\circ} \text { CONCEJ. } \\
\text { ELECTOS }\end{array}$ \\
\hline PARTIDO GALEGUISTA & 1.557 & 0 \\
PS de G - PSOE - & 9.616 & 7 \\
LOS VERDES DE GALICIA & 1.000 & 0 \\
PSG-EG & 521 & 0 \\
BNG & 2.880 & 2 \\
CDS & 601 & 0 \\
PP & 16.231 & 12 \\
ESQUERDA UNIDA & 2.298 & 1 \\
CNG & 3.926 & 3 \\
\hline
\end{tabular}


PROVINCIA:

J. ELECTORAL DE ZONA: MADRID

MUNICIPIO:

\section{MADRID}

\section{MADRID}

\begin{tabular}{lc}
\hline N. electores: & 2.512 .702 \\
N. ${ }^{\circ}$ votantes: & 1.493 .280 \\
N. ${ }^{\circ}$ votos de candidatura: & 1.470 .042 \\
N. ${ }^{\circ}$ votos en blanco: & 18.052 \\
N. ${ }^{\circ}$ votos válidos: & 1.487 .761 \\
N. ${ }^{\circ}$ votos nulos: & 5.519 \\
\hline
\end{tabular}

\begin{tabular}{|l|c|c|}
\hline CandidATURAS & $\begin{array}{c}\text { VotOS } \\
\text { OBTENIDOS }\end{array}$ & $\begin{array}{c}\text { N. }^{\circ} \text { CONCEJ. } \\
\text { ELECTOS }\end{array}$ \\
\hline PSOE & 510.556 & 21 \\
PP & 702.834 & 30 \\
IU & 144.640 & 6 \\
\hline
\end{tabular}


PROVINCIA:

MÁLAGA

J. ELECTORAL DE ZONA: MÁLAGA

MUNICIPIO:

MÁLAGA

N. ${ }^{\circ}$ electores:

373.154

N. ${ }^{\circ}$ votantes:

178.741

N..$^{\circ}$ votos de candidatura:

176.272

N. ${ }^{\circ}$ votos en blanco:

N. ${ }^{\circ}$ votos válidos:

177.615

N. ${ }^{\circ}$ votos nulos:

901

\begin{tabular}{|l|r|c|}
\hline CANDIDATURAS & $\begin{array}{c}\text { VoTOS } \\
\text { OBTENIDOS }\end{array}$ & $\begin{array}{c}\text { N. }{ }^{\circ} \text { CONCEJ. } \\
\text { ELECTOS }\end{array}$ \\
\hline PSOE & 87.847 & 17 \\
NACIÓN ANDALUZA & 219 & 0 \\
CRISTIANOS PARA LA & 355 & 0 \\
DEMOCRACIA & 4.186 & 0 \\
LOS VERDES DE ANDALUCIA & 536 & 0 \\
UNIÓN POPULAR ANDALUZA & 46.169 & 8 \\
PP & 892 & 0 \\
SOLUCIÓN INDEPENDIENTE & 10.765 & 2 \\
PA & 2.675 & 0 \\
CDS & 22.628 & 4 \\
IU-CA & & \\
\hline
\end{tabular}


PROVINCIA:

MELILLA

J. ELECTORAL DE ZONA: MELILLA

MUNICIPIO:

MELILLA

N. ${ }^{\circ}$ electores:

36.162

N. ${ }^{\circ}$ votantes:

20.753

N. ${ }^{\circ}$ votos de candidatura:

20.502

N. ${ }^{\circ}$ votos en blanco:

185

N. ${ }^{\circ}$ votos válidos:

20.687

N. ${ }^{\circ}$ votos nulos:

66

\begin{tabular}{|l|c|c|}
\hline CANDIDATURAS & $\begin{array}{c}\text { VoTOS } \\
\text { OBTENIDOS }\end{array}$ & $\begin{array}{c}\text { N. }{ }^{\circ} \text { CONCEJ. } \\
\text { ELECTOS }\end{array}$ \\
\hline PSOE & 8.387 & 11 \\
PP & 8.738 & 12 \\
GRUPO INDEPENDIENTE & 634 & 0 \\
HISPANO BEREBER & & 2 \\
PARTIDO NACIONALISTA & 1.780 & 0 \\
DE MELILLA & 249 & 0 \\
PARTIDO VERTICE & 409 & 0 \\
CDS & & 305 \\
$\begin{array}{l}\text { PARTIDO COALICION } \\
\text { MELILLENSE-PARTIDO MELILLENSE }\end{array}$ & & \\
\hline
\end{tabular}


PROVINCIA:

MURCIA

J. ELECTORAL DE ZONA: MURCIA

MUNICIPIO: MURCIA

\begin{tabular}{lr}
\hline N. ${ }^{\circ}$ electores: & 236.986 \\
N. ${ }^{\circ}$ votantes: & 156.160 \\
N. ${ }^{\circ}$ votos de candidatura: & 153.560 \\
N. ${ }^{\circ}$ votos en blanco: & 1.327 \\
N. ${ }^{\circ}$ votos válidos: & 154.887 \\
N. ${ }^{\circ}$ votos nulos: & 812 \\
\hline
\end{tabular}

\begin{tabular}{|l|c|c|}
\hline CANDIDATURAS & $\begin{array}{c}\text { VoTOS } \\
\text { OBTENIDOS }\end{array}$ & $\begin{array}{c}\text { N. }{ }^{\circ} \text { CONCEJ. } \\
\text { ELECTOS }\end{array}$ \\
\hline LOS VERDES & 2.527 & \\
PSOE & 61.723 & 13 \\
CDS & 5.559 & \\
IU & 15.124 & 3 \\
PP & 63.301 & 13 \\
ARCOIRIS & 1.104 & \\
ASOCIACION POL. PRO E.L.M. & & \\
PALMAR & 618 & \\
PARTIDO MURCIANISTA & 3.604 & \\
\hline
\end{tabular}


PROVINCIA:

NAVARRA

J. ELECTORAL DE ZONA: PAMPLONA

MUNICIPIO:

PAMPLONA-IRUÑA

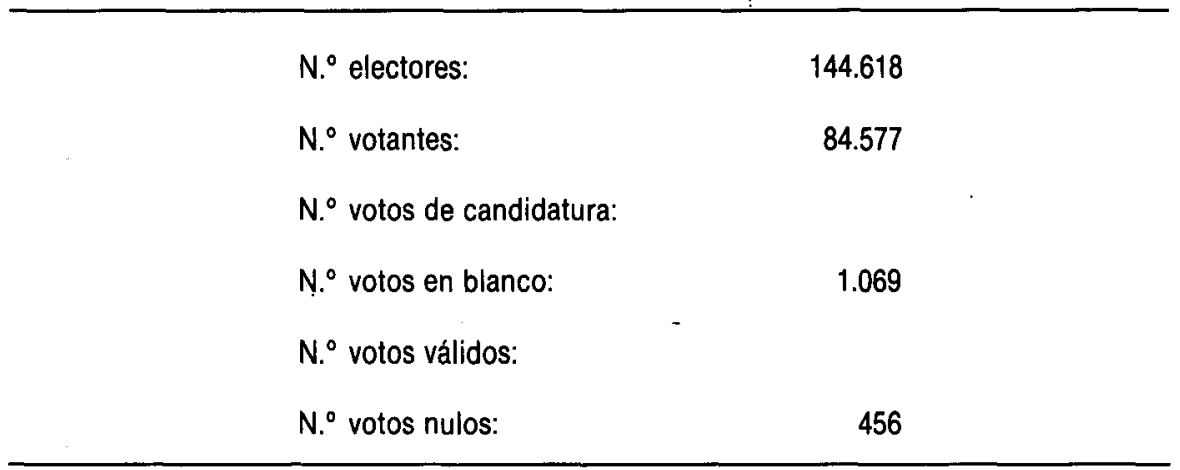

\begin{tabular}{|l|c|c|}
\hline CANDIDATURAS & $\begin{array}{c}\text { VoTOS } \\
\text { OBTENIDOS }\end{array}$ & $\begin{array}{c}\text { N. }{ }^{\circ} \text { CONCEJ. } \\
\text { ELECTOS }\end{array}$ \\
\hline HB & 10.835 & 4 \\
IU & 4.239 & 1 \\
EA & 4.418 & 1 \\
BATZARRE & 2.757 & \\
CDS & 1.838 & \\
EAJ-PNV & 751 & 13 \\
UPN & 32.820 & 8 \\
PSN-PSOE & 22.605 & \\
EE & 2.456 & \\
PARTIDO CARLISTA & 333 & \\
\hline
\end{tabular}


PROVINCIA:

ORENSE

J. ELECTORAL DE ZONA: ORENSE

MUNICIPIO: $\quad$ ORENSE

\begin{tabular}{lr}
\hline N. ${ }^{\circ}$ electores: & 85.454 \\
N. ${ }^{\circ}$ votantes: & 48.157 \\
N..$^{\circ}$ votos de candidatura: & 47.178 \\
N..$^{\circ}$ votos en blanco: & 507 \\
N..$^{\circ}$ votos válidos: & 47.685 \\
N. ${ }^{\circ}$ votos nulos: & 366 \\
\hline
\end{tabular}

\begin{tabular}{|l|c|c|}
\hline CANDIDATURAS & $\begin{array}{c}\text { VoTOS } \\
\text { OBTENIDOS }\end{array}$ & $\begin{array}{c}\text { N. }{ }^{\circ} \text { CONCEJ. } \\
\text { ELECTOS }\end{array}$ \\
\hline SI-OR & 1.347 & \\
BNG & 3.022 & 2 \\
EU & 1.413 & 12 \\
PS DE G-PSOE & 17.367 & 10 \\
FPG & 152 & 10 \\
PP & 15.114 & \\
PSG-EG & 614 & \\
ADEI-O & 726 & \\
LV & 198 & \\
CC E CDS & 5.441 & \\
PG & 1.654 & \\
LVEH & 130 & \\
\hline
\end{tabular}


PROVINCIA:

PALENCIA

J. ELECTORAL DE ZONA: PALENCIA

MUNICIPIO:

PALENCIA

N. ${ }^{\circ}$ electores:

58.399

N. ${ }^{\circ}$ votantes:

36.373

N. ${ }^{\circ}$ votos de candidatura:

35.362

$\mathrm{N} .^{\circ}$ votos en blanco:

683

N. ${ }^{\circ}$ votos válidos:

36.045

$N .^{\circ}$ votos nulos:

328

\begin{tabular}{|l|c|c|}
\hline CANDIDATURAS & $\begin{array}{c}\text { VotOS } \\
\text { OBTENIDOS }\end{array}$ & $\begin{array}{c}\text { N. }{ }^{\circ} \text { CONCEJ. } \\
\text { ELECTOS }\end{array}$ \\
\hline CDS & 1.885 & 1 \\
IU & 2.506 & 1 \\
PSOE & 14.145 & 11 \\
PP & 12.567 & 10 \\
UNIDAD PALENTINA & 891 & 0 \\
AGRUPACION PALENTINA & & 2 \\
\hline POPULAR & 3.368 & \\
\hline
\end{tabular}


PROVINCIA:

PONTEVEDRA

J. ELECTORAL DE ZONA: PONTEVEDRA

MUNICIPIO:

PONTEVEDRA
N. ${ }^{\circ}$ electores:
52.104
N. ${ }^{\circ}$ votantes:
30.829
N. ${ }^{\circ}$ votos de candidatura:
30.218
N. ${ }^{\circ}$ votos en blanco:
366
$N{ }^{\circ}$ votos válidos:
30.584
N. votos nulos:
245

\begin{tabular}{|l|r|c|}
\hline CANDIDATURAS & $\begin{array}{c}\text { VoTOS } \\
\text { OBTENIIOS }\end{array}$ & $\begin{array}{c}\text { N. }{ }^{\circ} \text { CONCEJ. } \\
\text { ELECTOS }\end{array}$ \\
\hline PP & 12.238 & 12 \\
CDS & 702 & 0 \\
PS de G-PSOE & 6.851 & 6 \\
PARTIDO GALEGUISTA & 80 & 0 \\
PLATAFORMA VECINAL DE & 98 & 0 \\
ESQUERDA & 305 & 0 \\
EU & 1.142 & 0 \\
PSG-EG & 3.261 & 3 \\
CNG & 4.135 & 4 \\
BNG & 1.406 & 0 \\
PONTEVEDRA UNIDA & & \\
\hline
\end{tabular}


PROVINCIA:

J. ELECTORAL DE ZONA: SALAMANCA

MUNICIPIO:
SALAMANCA

SALAMANCA
N. ${ }^{\circ}$ electores:

N. ${ }^{\circ}$ votantes:

N. ${ }^{\circ}$ votos de candidatura:

N. ${ }^{\circ}$ votos en blanco:

$N{ }^{\circ}$ votos válidos:

$N .^{\circ}$ votos nulos:
127.580

79.228

77.301

1.365

78.666

562

\begin{tabular}{|l|c|c|}
\hline CANDIDATURAS & $\begin{array}{c}\text { VoTOS } \\
\text { OBTENIDOS }\end{array}$ & $\begin{array}{c}\text { N. }{ }^{\circ} \text { CONCEJ. } \\
\text { ELECTOS }\end{array}$ \\
\hline LOS VERDES & 2.154 & 0 \\
PP & 34.375 & 13 \\
PRERAL & 315 & 0 \\
PSOE & 31.425 & 12 \\
DEMOCRACIA REGIONALISTA & 300 & 0 \\
IU & 4.081 & 1 \\
CDS & 4.650 & 1 \\
\hline
\end{tabular}


PROVINCIA:

\section{SEGOVIA}

J. ELECTORAL DE ZONA: SEGOVIA

MUNICIPIO: SEGOVIA

\begin{tabular}{lc}
\hline N. electores: & 42.045 \\
$N .^{\circ}$ votantes: & 25.166 \\
$\mathrm{~N}^{\circ}$ votos de candidatura: & 24.231 \\
$\mathrm{~N} .^{\circ}$ votos en blanco: & 612 \\
$\mathrm{~N} .{ }^{\circ}$ votos válidos: & 24.843 \\
$\mathrm{~N} .{ }^{\circ}$ votos nulos: & 323 \\
\hline
\end{tabular}

\begin{tabular}{|l|c|c|}
\hline CANDIDATURAS & $\begin{array}{c}\text { VOTOS } \\
\text { OBTENIDUS }\end{array}$ & $\begin{array}{c}\text { N. }{ }^{\circ} \text { CONCEJ. } \\
\text { ELECTOS }\end{array}$ \\
\hline PP & 10.163 & 11 \\
PSOE & 7.834 & 9 \\
CDS & 2.671 & 3 \\
IU & 2.600 & 2 \\
VERDES & 506 & 0 \\
PRC & 457 & 0 \\
\hline
\end{tabular}


PROVINCIA:

SEVILLA

J. ELECTORAL DE ZONA: SEVILLA

MUNICIPIO:

SEVILLA
N. electores:
514.077
$\mathrm{N}^{\circ}$ votantes:
281.224
$\mathrm{N} \cdot{ }^{\circ}$ votos de candidatura:
278.109
$N .{ }^{\circ}$ votos en blanco:
2.211
N. ${ }^{\circ}$ votos válidos:
280.320
N. ${ }^{\circ}$ votos nulos:
904

\begin{tabular}{|l|c|c|}
\hline CANDIDATURAS & $\begin{array}{c}\text { VotOS } \\
\text { OBTENIDOS }\end{array}$ & $\begin{array}{c}\text { N. }{ }^{\circ} \text { CONCEJ. } \\
\text { ELECTOS }\end{array}$ \\
\hline PP & 68.206 & 8 \\
PA & 77.168 & 9 \\
VERDES & 2.714 & 0 \\
FEJONS & 280 & 0 \\
PSOE-A & 108.028 & 12 \\
PST & 547 & 0 \\
UPAN & 216 & 0 \\
IU-CA & 19.216 & 2 \\
CDS & 877 & 0 \\
COALICION LOS VERDES LISTA & & 0 \\
ECOLOGISTA-HUMANISTA & 857 & \\
\hline
\end{tabular}


PROVINCIA:

J. ELECTORAL DE ZONA: SORIA

MUNICIPIO:
SORIA

SORIA
N..$\dot{e}$ electores:
25.352
N. ${ }^{\circ}$ votantes:
13.411
N. ${ }^{\circ}$ votos de candidatura:
12.925
$N .^{\circ}$ votos en blanco:
380
N. ${ }^{\circ}$ votos válidos:
13.305
N. ${ }^{\circ}$ votos nulos:
106

\begin{tabular}{|l|c|c|}
\hline CANDIDATURAS & $\begin{array}{c}\text { VotOS } \\
\text { OBTENIDOS }\end{array}$ & $\begin{array}{c}\text { N. }{ }^{\circ} \text { CONCEJ. } \\
\text { ELECTOS }\end{array}$ \\
\hline PSOE & 4.361 & 8 \\
PP & 6.485 & 12 \\
CDS & 902 & 1 \\
UC & 503 & 0 \\
IU & 389 & 0 \\
PANCAL & 195 & 0 \\
IC & 90 & 0 \\
\hline
\end{tabular}


PROVINCIA:

SANTA CRUZ DE TENERIFE

J. ELECTORAL DE ZONA: SANTA CRUZ DE TENERIFE MUNICIPIO:

SANTA CRUZ DE TENERIFE
$N 0^{\circ}$ electores:
160.264
$N .^{\circ}$ votantes:
72.046
$\mathrm{N} .{ }^{\circ}$ votos de candidatura:
70.528
$N^{\circ}{ }^{\circ}$ votos en blanco:
936
N. ${ }^{\circ}$ votos válidos:
71.464
N. ${ }^{\circ}$ votos nulos:
582

\begin{tabular}{|l|r|c|}
\hline CANDIDATURAS & $\begin{array}{c}\text { VotOS } \\
\text { OBTENIDOS }\end{array}$ & $\begin{array}{c}\text { N. }{ }^{\circ} \text { CONCEJ. } \\
\text { ELECTOS }\end{array}$ \\
\hline PSOE & 14.297 & 6 \\
PST & 393 & \\
ICAN & 5.241 & 2 \\
CDS & 1.859 & \\
LG & 416 & \\
PP & 7.576 & 3 \\
CI & 917 & \\
ATI & 39.053 & 16 \\
PLC & 776 & \\
\hline
\end{tabular}


PROVINCIA:

J. ELECTORAL DE ZONA: TERUEL

MUNICIPIO:

\section{TERUEL}

TERUEL
N. ${ }^{\circ}$ electores:
21.882
N..$^{\circ}$ votantes:
13.624
N. ${ }^{\circ}$ votos de candidatura:
13.348
N. ${ }^{\circ}$ votos en blanco:
182
N. ${ }^{\circ}$ votos válidos:
13.530
N. ${ }^{\circ}$ votos nulos:
94

\begin{tabular}{|l|c|c|}
\hline CANDIDATURAS & $\begin{array}{c}\text { VotOS } \\
\text { OBTENIDOS }\end{array}$ & $\begin{array}{c}\text { N. }{ }^{\circ} \text { CONCEJ. } \\
\text { ELECTOS }\end{array}$ \\
\hline INDEPENDIENTES POR TERUEL & 647 & \\
PARTIDO ARAGONES & 2.378 & 4 \\
PP & 3.948 & 7 \\
PSOE & 4.739 & 9 \\
CHUNTA ARAGONESISTA & 776 & 1 \\
CDS & 499 & \\
CONVERGENCIA ALTERNATIVA DE & 361 & \\
ARAGON-IU & & \\
\hline
\end{tabular}


PROVINCIA:

TOLEDO

J. ELECTORAL DE ZONA: TOLEDO

MUNICIPIO: TOLEDO
N. ${ }^{\circ}$ electores:
44.126
N. votantes:
27.732
N. ${ }^{\circ}$ votos de candidatura:
27.647
$\mathrm{N} .^{\circ}$ votos en blanco:
343
N. ${ }^{\circ}$ votos válidos:
28.090
$N^{\circ}$ votos nulos:
150

\begin{tabular}{|l|c|c|}
\hline CANDIDATURAS & $\begin{array}{c}\text { Votos } \\
\text { OBTENIDOS }\end{array}$ & $\begin{array}{c}\text { N. }{ }^{\circ} \text { CONCEJ. } \\
\text { ElECTOS }\end{array}$ \\
\hline PP & 12.272 & 12 \\
PSOE & 11.667 & 11 \\
IU & 2.948 & 2 \\
\hline
\end{tabular}


PROVINCIA:

TARRAGONA

J. ELECTORAL DE ZONA: TARRAGONA

MUNICIPIO:

TARRAGONA
N. ${ }^{\circ}$ electores:
84.975
N..$^{\circ}$ votantes:
51.458
$\mathrm{N} .{ }^{\circ}$ votos de candidatura:
50.982
$N .^{\circ}$ votos en blanco:
323
N. ${ }^{\circ}$ votos válidos:
51.305
$\mathrm{N} .{ }^{\circ}$ votos nulos:
153

\begin{tabular}{|l|c|c|}
\hline CANDIDATURAS & $\begin{array}{c}\text { VotOS } \\
\text { OBTENIDOS }\end{array}$ & $\begin{array}{c}\text { N. }{ }^{\circ} \text { CONCEJ. } \\
\text { ELECTOS }\end{array}$ \\
\hline CIU & 22.627 & 14 \\
PSC-PSOE & 17.844 & 11 \\
PP & 4.429 & 2 \\
AV & 1.960 & 0 \\
IC & 1.950 & 0 \\
ERC & 1.612 & 0 \\
CDS & 560 & 0 \\
\hline
\end{tabular}


PROVINCIA:

VALENCIA

J. ELECTORAL DE ZONA: VALENCIA

MUNICIPIO:

VALENCIA
N. ${ }^{\circ}$ electores:
591.157
N..$^{\circ}$ votantes:
375.043
$\mathrm{N} .^{\circ}$ votos de candidatura:
370.127
$\mathrm{N} .^{\circ}$ votos en blanco:
3.711
$\mathrm{N} .^{\circ}$ votos válidos:
373.838
N. ${ }^{\circ}$ votos nulos:
1.295

\begin{tabular}{|l|c|c|}
\hline CANDIDATURAS & $\begin{array}{c}\text { VotOS } \\
\text { OBTENIDOS }\end{array}$ & $\begin{array}{c}\text { N. }{ }^{\circ} \text { CONCEJ. } \\
\text { ELECTOS }\end{array}$ \\
\hline PSOE & 139.500 & 14 \\
UNIÓ VALENCIA & 80.500 & 8 \\
PARTIDO RADICAL SOCIALISTA & 878 & 0 \\
VALENCIANO & 7.774 & 0 \\
CDS & 95.238 & 9 \\
PP & 8.945 & 0 \\
LOS VERDES & 29.814 & 2 \\
ESQUERRA UNIDA DEL PAÍS & 5.982 & 0 \\
VALENCIÁ & 386 & 0 \\
UNITAT DEL POBLE VALENCIA & 628 & 0 \\
FALANGE ESPAÑOLA DE LAS JONS & 482 & 0 \\
FRENTE NACIONAL & & 0 \\
PLATAFORMA D'ESQUERRES & & 0 \\
\hline
\end{tabular}


PROVINCIA:

J. ELECTORAL DE ZONA: VALLADOLID

MUNICIPIO:

\section{VALLADOLID}

VALLADOLID
N. ${ }^{\circ}$ electores:
257.193
N. ${ }^{\circ}$ votantes:
155.021
N..$^{\circ}$ votos de candidatura:
150.379
N. ${ }^{\circ}$ votos en blanco:
2.870
N. ${ }^{\circ}$ votos válidos:
153.249
$\mathrm{N} .^{\circ}$ votos nulos:
1.353

\begin{tabular}{|l|c|c|}
\hline CANDIDATURAS & $\begin{array}{c}\text { VoTOS } \\
\text { OBTENIDOS }\end{array}$ & $\begin{array}{c}\text { N. }{ }^{\circ} \text { CONCEJ. } \\
\text { ELECTOS }\end{array}$ \\
\hline PP & 62.432 & 13 \\
PSOE & 58.204 & 12 \\
IU & 16.192 & 3 \\
CDS & 8.108 & 1 \\
PARTIDO DE LOS VERDES & 2.263 & \\
DEMOCR. REGIONALISTA CL & 1.374 & \\
COAL. VERDES ECOL. HUM. & 1.125 & \\
FE-JONS & 408 & \\
PLAT UNIT DE LA IZQUIERDA & 273 & \\
\hline
\end{tabular}


PROVINCIA:

\section{VIZCAYA}

J. ELECTORAL DE ZONA: BILBAO

MUNICIPIO:

BILBAO

N. ${ }^{\circ}$ electores:

312.032

N. ${ }^{\circ}$ votantes:

167.244

N. ${ }^{\circ}$ votos de candidatura:

164.370

$\mathrm{N} .{ }^{\circ}$ votos en blanco:

1.636

N. ${ }^{\circ}$ votos válidos:

166.006

$N{ }^{\circ}$ votos nulos:

1.238

\begin{tabular}{|l|c|c|}
\hline CANDIDATURAS & $\begin{array}{c}\text { VOTOS } \\
\text { OBTENIDOS }\end{array}$ & $\begin{array}{c}\text { N. }{ }^{\circ} \text { CONCEJ. } \\
\text { ELECTOS }\end{array}$ \\
\hline EAJ-PNV & 57.639 & 11 \\
EE & 11.504 & 2 \\
PSE-PSOE & 34.849 & 6 \\
HB & 20.180 & 4 \\
IU & 2.864 & \\
PARTIDO SOCIALISTA DE LOS & 447 & \\
TRABAJADORES & 23.436 & 2 \\
PP & 10.370 & \\
EA & 215 & \\
ALIANZA POR LA REPÚBLICA & 157 & \\
PARTIDO CARLISTA & 1.260 & \\
CDS & 1.449 & \\
EUSKAL HERRIKO BERDEAK & \\
\hline
\end{tabular}


PROVINCIA:

\section{ZAMORA}

J. ELECTORAL DE ZONA: ZAMORA

MUNICIPIO:

ZAMORA
$N{ }^{\circ}$ electores:
49.205
N. ${ }^{\circ}$ votantes:
27.753
N. ${ }^{\circ}$ votos de candidatura:
26.779
$\mathrm{N} .{ }^{\circ}$ votos en blanco:
622
N. ${ }^{\circ}$ votos válidos:
27.401
N..$^{\circ}$ votos nulos:
352

\begin{tabular}{|l|c|c|}
\hline CANDIDATURAS & $\begin{array}{c}\text { VoTOS } \\
\text { OBTENIDOS }\end{array}$ & $\begin{array}{c}\text { N. } .^{\circ} \text { CONCEJ. } \\
\text { ELECTOS }\end{array}$ \\
\hline IU & 1.714 & 1 \\
PSOE & 11.534 & 12 \\
PP & 11.397 & 11 \\
CDS & 1.790 & 1 \\
PARTIDO REGIONALISTA PAIS & & \\
\hline LEONÉS & 344 & \\
\hline
\end{tabular}


PROVINCIA:

J. ELECTORAL DE ZONA: ZARAGOZA

MUNICIPIO:
ZARAGOZA

ZARAGOZA
N. ${ }^{\circ}$ electores:
466.215
N. ${ }^{\circ}$ votantes:
263.294
$\mathrm{N} .{ }^{\circ}$ votos de candidatura:
$N .^{\circ}$ votos en blanco:
3.815
$N{ }^{\circ}$ votos válidos:
261.113
N. ${ }^{\circ}$ votos nulos:
1.794

\begin{tabular}{|l|c|c|}
\hline CANDIDATURAS & $\begin{array}{c}\text { VOTOS } \\
\text { OBTENIDOS }\end{array}$ & $\begin{array}{c}\text { N. }{ }^{\circ} \text { CONCEJ. } \\
\text { ELECTOS }\end{array}$ \\
\hline PSOE & 112.106 & 15 \\
PP & 56.947 & 7 \\
PAR & 47.780 & 6 \\
CAA-IU & 24.594 & 3 \\
CDS & 7.499 & \\
CHA & 6.082 & \\
PARTIDO SOCIALISTA DE LOS & & \\
TRABAJADORES & 1.574 & \\
MOVIMIENTO ARAGONES SOCIAL & 716 & \\
\hline
\end{tabular}


EVOLUCIÓN DEL VOTO EN LAS ELECCIONES MUNICIPALES 6

\begin{tabular}{|c|c|c|c|c|c|c|c|c|c|}
\hline \multirow{2}{*}{ Partidoos } & \multicolumn{3}{|c|}{ MUNICIPALES 1991} & \multicolumn{3}{|c|}{ LEGISLATIVAS 1989} & \multicolumn{3}{|c|}{ MUNICIPALES 1987} \\
\hline & Concejales & $\%$ & N. ${ }^{\circ}$ Votos & Diputados & $\%$ & N..$^{\circ}$ Votos & Concejales & $\%(2)$ & N. ${ }^{\circ}$ Votos \\
\hline PSOE & 22.904 & 38,40 & 7.163 .668 & 175 & 39,63 & 7.713 .810 & 23.241 & 36,74 & 7.229 .782 \\
\hline$P P$ & 15.740 & 25,21 & 4.703 .468 & 107 & 25,40 & 4.945 .134 & 16.581 & 20,74 & 4.080 .705 \\
\hline IU & 2.600 & 8,46 & 1.578 .361 & 17 & 9,21 & 1.791 .925 & 2.268 & 6,16 & 1.212 .262 \\
\hline CDS & 2.568 & 3,87 & 721.832 & 14 & 7,84 & 1.525 .536 & 5.952 & 9,67 & 1.902 .293 \\
\hline $\mathrm{CiU}$ & 3.690 & 4,85 & 905.410 & 18 & 5,30 & 1.032 .243 & 4.350 & 5,10 & 1.004 .115 \\
\hline PNV & 951 & 1,60 & 298.766 & 5 & 1,31 & 254.681 & 819 & 1,23 & 241.832 \\
\hline $\mathrm{HB}$ & 677 & 1,06 & 198.445 & 4 & 1,12 & 217.278 & 669 & 1,21 & 239.010 \\
\hline PA & 536 & 1,83 & 341.137 & 2 & 1,09 & 212.687 & 294 & 1,13 & 221.825 \\
\hline PAR & 768 & 0,65 & 120.643 & 1 & 0,37 & 71.733 & 896 & 0,66 & 129.370 \\
\hline UV (1) & 334 & 1,01 & 187.535 & 2 & 0,74 & 144.924 & 215 & 0,76 & 148.878 \\
\hline EA & 381 & 0,70 & 131.087 & 2 & 0,70 & 136.955 & 497 & 1,05 & 207.054 \\
\hline$E E$ & 105 & 0,38 & 71.442 & 2 & 0,54 & 105.238 & 157 & 0,55 & 107.354 \\
\hline $\mathrm{AiC}$ & 281 & 0,75 & 140.261 & 1 & 0,33 & 64.767 & - & (3) & - \\
\hline
\end{tabular}

(1) Unión Valenciana. (2) Sobre el voto válido. (3) No existía en 1987.

$\stackrel{\omega}{\exists}{ }_{6}^{6}$ El País, 28 de mayo de 1991. 


\section{ANÁLISIS GENERAL DE LOS RESULTADOS}

EI PSOE logra la mayoria absoluta en las Comunidades Autónomas de Castilla-La Mancha, Extremadura, Murcia y Valencia; la mayoría relativa -como lista más votada - en Asturias, Aragón, Canarias, Cantabria y La Rioja. El PP logra la mayoría absoluta en las Comunidades de Baleares y Castilla-León; la relativa en Madrid y Navarra (PP-UPN).

En las elecciones municipales -en las capitales de provincia- el PSOE llega a la mayoria absoluta en Albacete, Badajoz, Cádiz, Coruña, Cuenca, Geroná, Huelva, Lérida y Málaga; es la lista más votada en Almería, Barcelona, Ciudad Real, Huesca, Las Palmas, Orense, Palencia, SeviIla, Teruel, Valencia, Zamora y Zaragoza. El PP logra mayoria absoluta en Madrid, Palma de Mallorca (PP-UM), Burgos, Castellón y Soria; es el partido más votado en Ávila, Granada, Guadalajara, Navarra (UPN-PP), León, Logroño, Lugo, Melilla, Oviedo, Pontevedra, Salamanca, Segovia, Toledo y Valladolid.

EI PP y el PSOE logran igual número de concejales en Alicante, Cáceres, Jaén y Murcia, aunque es más votado el PSOE en la primera ciudad y el PP en las otras tres. A su vez, el PSOE empata con la Unión para el Progreso de Cantabria, en Santander, en cuanto al número de concejales pero es más votada la candidatura socialista.

A su vez, IU logra la mayoría relativa en Córdoba. Entre los partidos nacionalistas y regionalistas CiU tiene mayoría absoluta en Tarragona, PNV es la lista más votada en Bilbao y Vitoria y EA en San Sebastián; ATI llega a la mayoria absoluta en Tenerife y el Partido del Progreso y Futuro de Ceuta logra la mayoria absoluta en esta ciudad. Finalmente, el CDS desaparece prácticamente de las instituciones.

EI PSOE sigue siendo el partido más votado, aunque con un moderado descenso; avanza en las zonas rurales y en las Comunidades Autónomas más débiles y, sin embargo, pierde votos de aquella parte del electorado mejor informado ${ }^{7}$. Si bien se le van votos hacia la formación de Anguita, logra los votantes progresistas del CDS.

Su retroceso, con las pérdidas de las alcaldias de importantes ciudades, es más simbólico que matemático a la hora de computar los votos; sin embargo ello supone un indicio de cambio y una advertencia a tener en cuenta. Pese a este descenso moderado, en numerosas instituciones precisa del apoyo de IU para poder gobernar, lo que no dejará de ser pro-

7 Ver Diario 16, de 28 de mayo de 1991. C. DAviLA. Análisis electoral, y El Mundo, de 28 de mayo de 1991. F. Bocos. Análisis electoral. 
blemático y puede suponer una confrontación entre la política estatal y la autonómica o municipal, debido a los planteamientos más radicales de $\mathrm{IU}^{8}$.

EI PP consolida el voto urbano y logra un moderado avance, pese al cual se mantiene la diferencia de puntos existente entre este partido y el PSOE. Obtiene algunas victorias con gran valor simbólico en las alcaldias de Madrid, Sevilla y Valencia, que generan gran optimismo y respaldan, como eficaz, la gestión de José M. ${ }^{a}$ Aznar, que se consolida como líder capaz de evitar las disputas internas - tan nefastas en la derecha española- y de convertirse, aún a largo plazo, en alternativa del PSOE. EI PP es el gran beneficiado del desastre sufrido por el CDS, absorbiendo, en mayor medida que el PSOE, el espacio centrista, tan deseado por Aznar, y eliminando a un competidor; bien es verdad que en la decisión del electorado de Adolfo Suárez, parece que han pesado más los errores de éste que la oferta del $\mathrm{PP}^{9}$.

El ascenso electoral de IU supone la eficacia del mensaje renovador de Julio Anguita, quien se beneficia de los votantes desencantados del PSOE. Su ascenso es limitado pero importante, en cuanto que pierde su marginación y entra en la gran mayoría de las instituciones, teniendo, en numerosas ocasiones, la llave del gobierno municipal o autonómico. El éxito electoral de IU consolida el liderazgo de Anguita, en tiempos difíciles para la izquierda que él representa; su papel de azote de la corrupción política cala en este sector de la población.

El desastre electoral del CDS supone una variación del mapa político existente hasta el momento, con dos partidos mayoritarios y una segunda opción de cada electorado ${ }^{10}$. Tal fracaso sólo puede explicarse por el cúmulo de errores políticos de Adolfo Suárez, que los asume con su dimisión; sus pactos con el PP para llevar a cabo numerosas mociones de censura contra alcaldes socialistas -el caso de Madrid fue el más llamativo- aleja a los votantes más progresistas; a su vez, la posterior alianza con el PSOE a partir de 1989, en lugar de actuar como oposición crítica, alejó a los más moderados ${ }^{11}$ : sus votantes, pues, quedaron reducidos a esa mínima parte que, ya sin ninguna fuerza, le respaldó el 26 de mayo. Estas estrategias contradictorias, llevadas a cabo de forma personal por el propio Suárez, no fueron explicadas ni a su electorado ni a sus militantes. El resultado es conocido de todos: el CDS, si pervive, se convierte, de momento, en partido extrainstitucional. Dentro de las Comunidades Autónomas, sólo se mantiene en Asturias, Canarias, Castilla-León y Extremadura, aunque también con un fuerte descenso.

8 Ver Diario 16, de 28 de mayo de 1991. J. GonzAlez Ferrari. Análisis electoral.

9 Ver El Mundo, de 27 de mayo de 1991. C. Alvarez de Toledo. P. Altares.

10 Ver El País, 28 de mayo de 1991. Editorial.

11 Ver El Mundo, 27 de mayo de 1991. R. Pl; Diario 16, 16/27 de mayo de 1991. J. LACALLE. 
En el ámbito municipal, los Ayuntamientos se constituyen el 15 de junio, con la excepción de 10 -entre las capitales de provincia- que lo hacen el 5 de julio, debido a la presentación de recursos por fraude electoral: Alicante, Badajoz, Barcelona, Ciudad Real, Córdoba, Las Palmas, Madrid, Salamanca, Santa Cruz de Tenerife y Valencia. Junto al fraude habitual aumenta, de forma escandalosa, el fraude por correo, que afecta fundamentalmente al PP en la provincia de Salamanca ${ }^{12}$; este tipo delictivo pone de manifiesto la necesidad de una modificación de la normativa.vigente sobre este aspecto, que garantice la utilización adecuada de este medio electoral ${ }^{13}$.

Un dato importante a considerar es el fuerte ascenso de la abstención, cercana al 40 por 100 , sobre todo en el cinturón industrial de las grandes ciudades. Ello supone una variación comparativa, ya que en las elecciones autonómicas $y$, sobre todo, municipales, en que se plantean los problemas que afectan más directamente al ciudadano, la abstención suele disminuir respecto a las generales, términos que se invierten en esta convocatoria.

El análisis de este hecho lleva a las siguientes explicaciones, eliminando, al menos como definitiva, la del buen tiempo reinante, que se sumará en tal caso a la apatía del electorado que responde a causas más profundas.

En primer lugar, hay que entender la abstención como un rechazo importante ante los escándalos políticos aireados por la prensa en los últimos tiempos, que afectaron fundamentalmente al partido gobernante, que fue en suma, el más perjudicado por la ausencia de un importante número de votantes. Ello llevó a la pérdida para el PSOE de ciudades importantes con un fuerte cinturón industrial que se alejó de las urnas.

En segundo lugar, y un poco consecuencia de lo primero, el rechazo ante los mismos candidatos, dada la minima renovación de las listas electorales, es un hecho, llevado a un extremo esperpéntico en Marbella, con la elección del independiente y populista Gil y Gil.

Por último, el alto nivel de abstención coincide con el aumento del censo electoral, en más de un millón de jóvenes; su apatía ante un derecho esencial del ciudadano manifiesta el poco aprecio por el sistema político existente.

Por tanto, en estas elecciones se pueden señalar dos correctivos importantes a la tendencia anterior del electorado: el alto índice de abstención y la pérdida para el PSOE de alcaldias en ciudades clave.

12 No sólo a la capital de la provincia, sino a 329 Corporaciones de las 362 totales.

13 Ver El Sol, 14 de junio de 1991 y El Mundo, 15 de junio de 1991, J. DE EsTEBAN: «El voto por correo". 


\section{PACTOS POSTELECTORALES}

Los resultados electorales muestran claramente la necesidad de pactos, tanto en el ámbito autonómico como municipal, a fin de lograr la estabilidad y gobernabilidad de las instituciones.

Desde el mismo momento de conocerse los resultados comienza una segunda etapa de negociaciones y compromisos políticos en los que el elector no interviene directamente, aunque sí puede pedir responsabilidades a posteriori, como se puede observar en los resultados negativos del CDS; es un hecho comprobado que la desviación de la voluntad popular manifestada en las urnas, suele pasar factura en la siguiente consulta. El cuerpo electoral entiende y admite los compromisos políticos entre ideologías afines, pero rechaza todo pacto oportunista o carente de dignidad ${ }^{14}$.

Pese a la necesidad del pacto, dado el gran número de mayorias minoritarias existente, hay que tener presente el riesgo que conlleva el simple reparto de ámbitos de poder generador de estabilidad o de fisuras y resentimientos. Es importante a la hora de las negociaciones el mantener el equilibrio entre los dos elementos del binomio estabilidad-representatividad; en este sentido puede considerarse lo siguiente:

a) Ambos elementos alcanzan el máximo nivel cuando un partido logra la mayoria absoluta.

b) Si ésta no existe, será precisa una coalición, ya que los gobiernos en minoria tienen un alto riesgo de inestabilidad y, en consecuencia, de ineficacia.

c) Cuando forma parte de la coalición el partido más votado hay un alto grado de representatividad y si los partidos aliados son afines y forman una mayoría justa, la estabilidad será muy aceptable; hay que tener en cuenta que el riesgo de enfrentamiento aumenta en proporción directa al número de socios de la coalición.

d) Por el contrario, en el caso de que la coalición que accede a las instituciones de gobierno no integre al partido más votado, puede lograrse estabilidad aunque disminuye la representatividad, disminución que se modera si existe afinidad entre las partes aliadas ${ }^{15}$.

14 Ver $A B C$, de 27 de mayo de 1991 y Diario 16, de 28 de mayo de 1991

15 Ver, en este sentido, el trabajo del Equipo de análisis político de la Universidad Autónoma de Barcelona, publicado en El Pais, el 21 de junio de 1991. 
El eje en torno al que han girado las negociaciones y los compromisos políticos concretos ha sido el de mantener la eficacia en la gestión, autonómica o municipal, dato al que es más sensible el ciudadano que a la discusión sobre el modelo de sociedad, que ya no es el problema español ${ }^{16}$. Pese al cumplimiento general de esta idea hay que señalar la existencia de excepciones, tanto en el sentido de pactos que han dado el Gobierno - municipal o autonómico- a un partido distinto al más votado, como compromisos generadores de crisis políticas o algún supuesto realmente escandaloso para la opinión pública. Sin embargo, al ser estos hechos minoritarios, no pueden descalificar globalmente la eficacia de los pactos que, parece, han generado una importante estabilidad en las instituciones. Veámoslo por separado.

a) En primer lugar, han existido compromisos políticos que han dado el Gobierno a un partido distinto al más votado, lo que redunda en perjuicio de la representatividad, como ya se ha señalado.

En el ámbito autonómico éste ha sido el caso de las Comunidades Autónomas de Aragón y Cantabria -en beneficio del PP- y Madrid -en beneficio del PSOE- De 13 Comunidades Autónomas, seis han tenido mayoria absoluta de un solo partido; en cuatro se ha respetado la lista más votada (Asturias, Canarias, Navarra y Rioja) y solamente en tres la coalición ganadora no integra al partido con mayoría minoritaria (Aragón, Cantabria y Madrid), sin embargo, sí se ha pactado entre partidos afines ideológicamente ${ }^{17}$, aunque en Cantabria, como se verá, con grave escándalo de la opinión pública.

Respecto al ámbito municipal, y considerando solamente las capitales de provincia, se pueden señalar varios casos de coaliciones en contra de la lista más votada. Así, Cáceres, Jaén, Logroño, Murcia, Salamanca, San Sebastián, Toledo y Valladolid -en beneficio del PSOE-; Las Palmas, Santander y Valencia -en beneficio del PP-; Teruel y Sevilla -en favor de partidos regionalistas-, y Guadalajara de IU.

Dentro de todos estos supuestos hay que señalar.-por curioso-el caso de la alcaldía de Guadalajara en la que, sin pacto y por evitar el gobierno de la derecha - lista más votada-, el PSOE - con 10 concejalesante la negativa de IU a apoyar al concreto equipo municipal socialista, da sus votos sorpresivamente a IU, logrando este partido -con sólo tres concejales - la alcaldía de la ciudad.

b) En segundo lugar, determinados pactos han originado crisis po-

16 Ver El Pais, El Mundo, ABC, Diario 16 y El Sol, de los dias 27 y 28 de mayo, 14 y 15 de junio de 1991.

17 Aragón: PP-PAR. 
líticas por ser, en cierto modo, contra natura ${ }^{18}$; estos supuestos sólo se han dado en el ámbito municipal.

En San Sebastián la lista más votada es la de EA, pero el alcalde pertenecerá al PSOE debido al pacto alcanzado con el PNV; el resentimiento de EA, que prima emocionalmente sobre las ideas políticas, le hará abstenerse en la votación de determinadas corporaciones, dando entrada en varios equipos municipales a $\mathrm{HB}$, en contra del Pacto de Ajuria Enea.

Esta nueva alianza del PNV pone en grave riesgo la estabilidad del gobierno tripartito nacionalista (PNV, EA, EE), con la consiguiente ruptura ocurrida a los dos meses. Sin embargo, en el momento postelectoral, el PNV se alia con el PSOE en un pacto global para formar mayorias en Diputaciones y Ayuntamientos, ya que el compromiso con sus socios de gobierno autonómico -EA y EE - no le permite llegar a mayorias capaces de gobernar en las instituciones locales, perjudicando de manera importante las perspectivas políticas de estos partidos. Así, el Alcalde de San Sebastián será socialista mientras que el Presidente de la Diputación pertenecerá al PNV. El Lehendakari Ardanza, principal impulsor del pacto nacionalista, queda en difícil situación política ante la nueva alianza dirigida por el Presidente del PNV Arzallus. Las consecuencias se verán pocos meses después ${ }^{19}$.

Otro caso singular es el de la alcaldía de Sevilla, lograda por el andalucista Rojas Marcos con el apoyo del PP, contra la lista más votada del PSOE, con el fin de impedir la gestión socialista en su ciudad madre. El PA y el PP se reparten las competencias municipales al 50 por 100 , quedando Rojas Marcos como Alcalde (PA) y Soledad Becerril como Primera Teniente Alcalde (PP), con coordinación sobre las competencias que corresponden a su partido ${ }^{20}$. El Presidente del PA -P. Pacheco- dimite al considerar que, a largo plazo, este extraño pacto perjudicará a su partido.

En Canarias se pueden señalar compromisos que, pese a no afectar a capitales de provincia, son tan chocantes que merecen ser reseñados; se llega a la alianza del PP con el PSOE a fin de arrebatar determinadas alcaldias a partidos regionalistas: ATI, AHI o ICAN. Así, en La Laguna, el PSOE logra la alcaldia con el apoyo del PP, pese a que ATI fue la lista más

\footnotetext{
Cantabria: PP-UPPC.

Madrid: PSOE-IU.

18 Ver EI Sol, El País y Diario 16, de 18 de junio de 1991.

19 En septiembre de este año se llega a la ruptura del Pacto de gobierno autonómico PNV-EA-EE, iniciando el PNV un nuevo compromiso con el PSOE; el Gobierno tomará posesión en octubre.

${ }_{20}$ Según la eficaz fórmula seguida en Madrid, tras la moción de censura que derrocó al socialista Barranco, entre Rodríguez Sahagún (CDS) y Álvarez del Manzano (PP).
} 
votada. Igualmente sucede en las capitales de las islas de La Palma y de Hierro: en la primera, la alcaldía es para el PP — con el apoyo del PSOEpese a la mayoria minoritaria de ICAN; en Hierro es para el PSOE - con el apoyo del PP - siendo AHI la lista más votada.

c) Finalmente, el caso de Cantabria supone un escándalo general en la opinión pública ${ }^{21}$ ante el pacto entre el PP y la plataforma electoral creada por el polémico Hormaechea con personas procedentes, en su mayoria, de las filas de Aznar.

La UPPC logra 10 concejales frente a los seis del PP; pese a que hace seis meses el PP se unió al PSOE para derrocar al Presidente del Ejecutivo cártabro, Hormaechea, por indignidad política, formando ambos partidos un gobierno autonómico de gestión, ahora han de dar la espalda a sus pasadas actuaciones -en pro del honor-si no quieren dejar las instituciones en manos del PSOE (lista más votada en la Autonomía y en la capital). El pacto - global para la Comunidad-se hará bajo la condición de que la formación de Hormaechea se integre en el PP - de donde procede-; la alcaldía de Santander será para este partido y la presidencia del Ejecutivo nuevamente - mor politicae-para Hormaechea.

\section{ANÁLISIS DE LOS RESULTADOS AUTONÓMICOS EN LAS 13 COMUNIDADES ${ }^{22}$}

\section{Aragón}

EI PSOE es la lista más votada, con 30 escaños, tres más que en 1987; a su vez, IU consigue otro diputado, logrando tres escaños. EI PP sube de 13 a 17 escaños mientras que el PAR baja de 19 a 17. EI CDS desaparece de la Asamblea, repartiéndose sus seis diputados anteriores entre el PSOE y el PP. Pese al mayor voto socialista, la coalición PP-PAR logra la mayoría absoluta, pudiendo repetir gobierno; el PAR accede a la presidencia del Ejecutivo aragonés mientras que el PP controla las competencias económicas y preside el Parlamento Autonómico. La abstención supera el 35 por 100.

21 Ver El País, Diario 16, El Sol y El Mundo, de los días 14 y 15 de junio de 1991.

22 Ver Diario 16, El País y El Sol, de 28 de mayo de 1991. 


\section{Asturias}

EI PSOE logra la mayoria relativa con 21 escaños (uno más que en 1987); se observa un moderado ascenso del PP, que gana dos diputados (pasa de 13 a 15) y de IU que logra otros dos (de cuatro a seis). EI CDS sufre el generalizado revés electoral, descendiendo desde ocho escaños a dos. Lo más relevante de esta Comunidad es el logro de representación por una coalición nacionalista (PAS-UNA), con un escaño, formada por los partidos Asturianista y Unidad Nacionalista Astur, que hasta ahora se habian presentado en listas separadas. La abstención - la más elevadasupera el 40 por 100.

Los seis escaños perdidos por el CDS han beneficiado al PP y también al PSOE. Este partido, pese a que gana un escaño, ha perdido votantes que absorbe IU y ha sido castigado por la abstención, lógico en una formación política que lleva gobernando tres legislaturas; sin embargo, el descalabro del CDS le ha permitido superar el previsible abandono de algunos votantes e, incluso, obtener un diputado más.

EI PSOE puede gobernar la Comunidad con el apoyo de IU; aunque el distinto enfoque de ambos partidos a la difícil situación económica de la región, pendiente de una segunda reconversión industrial, puede crear dificultades.

\section{Baleares}

La coalición electoral PP-UM, con 31 escaños (seis más que en 1987) logra la mayoría absoluta; el PSOE mantiene sus 21 diputados y el CDS pierde los cinco que tenía, siendo absorbidos por la coalición ganadora; también IU sufre un importante revés al perder los dos escaños logrados en 1987, siendo su mayor fracaso electoral en toda España. EI PSM desciende de cuatro a tres diputados. Obtiene representación por vez primera Entesa d'Esquerra de Menorca, con dos escaños, se mantiene -mínimamente- Federació d'Independents de Eivissa i Formentera y aparece Unió d'Independents de Mallorca; es decir, varios partidos regionales se sientan en el Parlamento autonómico. También en esta Comunidad es muy alto el índice de abstención, acercándose al 40 por 100 y afectando fundamentalmente a los partidos de izquierda. 


\section{Canarias}

También en esta autonomía ha sido el PSOE el partido más votado, logrando 23 escaños frente a los 21 que tenía anteriormente; el PP, sin embargo, se mantiene en los seis diputados anteriores y el CDS desciende bruscamente de 13 a siete. Numerosos partidos regionalistas mantienen representación en el Parlamento Autónomo: son de destacar los 16 diputados de la AIC (tenía 11) y los cinco de IC-IU (dos escaños en 1987); sin embargo, descienden de tres a dos la Asamblea Majorera (AM) y de dos a uno la Agrupación Herrereña Independiente (AHI). La abstención es alta, alrededor del 38 por 100 . La sangría de votos del CDS serán absorbidos por el PSOE y por AIC, no beneficiando aqui a las filas de Aznar. Las múltiples minorias posibilitan varios tipos de pactos, decidiéndose al fin el del PSOE y AIC, siendo socialista el Presidente del Ejecutivo.

\section{Castilla-La Mancha}

EI PSOE logra la mayoría absoluta por tercera vez, aumentando en dos los escaños que tenían en 1987; asciende también el PP en un escaño (19) e IU logra entrar en el Parlamento Autónomo. El CDS pierde los cuatro diputados que tenia, beneficiando a ambos partidos mayoritarios. Castilla-La Mancha es una de las Autonomías con menor índice de abstención, alrededor de 27 por 100 .

\section{Castilla-León}

Mayoría absoluta del PP con 43 escaños, lo que supone un considerable avance respecto a los 32 que logró en 1987; se beneficia claramente del desastre sufrido por el CDS, que pierde 13 diputados, quedando con una exigua representación de cinco en la Autonomía en que más escaños había logrado en 1987.

IU logra entrar en la Asamblea, con un diputado y el PSOE sube de 32 a 35 escaños, también a costa del CDS en buena parte. Si hasta ahora el PP había gobernado coaligado con el CDS, los votos procedentes de esta formación manifiestan igual voluntad electoral. La abstención, aceptable comparativamente, roza el 32 por 100. 


\section{Cantabria}

EI PSOE es el partido más votado, logrando 16 escaños (tres más que en 1987) y, a continuación, la singular plataforma electoral fundada por el discutido Hormaechea -UPPC- con 15 escaños, procedentes del PP; esta formación, en consecuencia, sufre un importante revés electoral, descendiendo de 18 a seis escaños, que le pone en una situación muy difícil para gobernar la Comunidad. EI PRC desciende también considerablemente desde sus cinco escaños anteriores a sólo dos, e IU no logra representación en la Asamblea.

El pacto PP-UPCA, muy criticado ${ }^{23}$, da la alcaldia de Santander al PP y la presidencia de la Comunidad a Hormaechea, quien integra su formación en el PP. Abstención cercana al 28 por 100, la más baja (junto a Castilla-La Mancha) de estos comicios, muy acorde con las especiales circunstancias politicas de esta Comunidad.

\section{Extremadura}

Mayoría absoluta socialista por tercera vez consecutiva, con 39 escaños (cinco más que en 1987). También avanza el PP, pasando de 17 a 19 diputados; ambas formaciones se reparten los cinco escaños que pierde el CDS (tres diputados de cinco que logró en 1987).

Éxito electoral, igualmente, de IU que aumenta en dos su número de escaños, quedando con cuatro; desastre también para la EU que pierde los cuatro diputados que tenía y sale del Parlamento autónomo. Abstención del 29 por 100 aproximado, una de las más bajas de estos comicios junto con Castilla-La Mancha y Cantabria.

\section{Madrid}

La lista más votada es la del PP, con 47 escaños frente a los 32 que tenía, siendo uno de los éxitos electorales de la formación de Aznar. EI PSOE se mantiene y logra un escaño más - 41 - pese al desgaste del equipo de Leguina; sube considerablemente IU, de siete a 13 escaños, y desaparece el CDS de la Asamblea, beneficiando fundamentalmente al PP, aunque tam-

23 Ver epígrafe 4. 
bién al PSOE, que compensa la pérdida de votos que han ido claramente a IU. Pese al éxito.electoral del PP, el gobierno de la Comunidad quedará nuevamente en manos socialistas ante el pacto PSOE-IU, aunque este pacto conlleva un control permanente de IU sobre la política del Presidente Leguina.

\section{Murcia}

Mayoria absoluta del PSOE pese a perder un escaño; subida moderada del PP, de 16 a 17 diputados y desaparición del CDS; sus tres escaños pasarán al PP y al PSOE, equilibrando los votos que esta formación pierde en favor de IU, que logrará cuatro escaños frente al único que adquirió en 1987. Los regionalismos no lograrán entrar en el Parlamento autonómico.

\section{Navarra}

La lista más votada será la de la unión PP-UPN, con 20 diputados; este resultado tiene una doble lectura: un éxito en cuanto al número que cada una de estas formaciones tenía anteriormente (12 y 14 escaños respectivamente) y un descenso en cuanto que el número logrado actualmente es bastante menor que la suma de cada uno de los socios; es decir, una parte de cada electorado no ha admitido la unión.

También el PSOE avanza considerablemente desde 15 a 19 escaños. El CDS desaparece en esta Comunidad y, por el contrario, IU logra representación en el Parlamento Autonómico, con dos diputados. Los avances señalados no sólo proceden de los votos del CDS sino también del general descenso nacionalista en esta Comunidad: HB baja de siete a seis escaños, EA de cuatro a tres, EE pierde el único diputado que tenía y el PNV tampoco tendrá representación en esta convocatoria.

Logrará el gobierno la unión PP-UPN, en quinta vuelta como lista más votada, según establece el Estatuto Navarro; sin embargo gobernará en minoría en un dificilísimo equilibrio político. Fuerte abstención, rondando el 40 por 100. 


\section{La Rioja}

Nuevamente es el PSOE el partido más votado, aumentando en dos el número de diputados (16); igualmente, el PP logra otros dos (15); el aumento de votos de ambos partidos es, a todas luces, procedente del CDS que pierde los cuatro escaños que tenia. Tampoco IU logra representación autonómica y el PR se mantiene en los dos escaños que ocupa desde 1983. El Gobierno queda en manos del PSOE con el apoyo, de nuevo, del PR. Abstención moderada, sobre el 30 por 100.

\section{Comunidad Valenciana}

EI PSOE logra la mayoria absoluta -45 diputados- que había perdido en 1987, lo que le supone gobernar cómodamente, sin necesidad de pactos. Importante ascenso del PP con siete diputados más (31) y ascenso prudente de UV en un escaño (7); estas tres formaciones se benefician, en mayor o menor medida del derrumbe electoral del CDS, que pierde los 11 escaños que tenia desde 1987; IU mantiene sus seis diputados. Abstención aceptable en esta Comunidad, alrededor del 30 por 100. 
PACTOS AUTONOMICOS

\begin{tabular}{|l|l|l|}
\hline COMUNIDAD & PARTIDO & PACTO \\
\hline Baleares & PP-UM & \\
Castilla-León & PP & \\
Castilla-La Mancha & PSOE & \\
Extremadura & PSOE & \\
Murcia & PSOE & \\
Valencia & PSOE & \\
Asturias & PSOE & IU \\
Aragón & PP & PRA \\
Cantabria & PP & UPPC \\
Canarias & PSOE & AIC \\
Madrid & PSOE & IU \\
Navarra & UPN-PP & \\
Rioja & PSOE & PR \\
\hline
\end{tabular}




\section{DISTINTAS ALCALDIAS EN LAS CAPITALES DE PROVINCIA}

PSOE: $27 \quad$ (Almería, Cádiz, Granada, Huelva, Jaén, Málaga, Huesca, Zaragoza, Albacete, Cuenca, Toledo, Ciudad Real, Palencia, Valladolid, Zamora, Salamanca, Lérida, Gerona, Barcelona, Alicante, Cáceres, Badajoz, La Coruña, Orense, Logroño, Murcia, San Sebastián)

PP: $\quad 15 \quad$ (Oviedo, Baleares (PP-UM), Santander, Avila, Burgos, León, Segovia, Soria, Castellón, Valencia, Lugo, Pontevedra, Navarra (UPN-PP), Melilla, Madrid)

PNV: $\quad 2 \quad$ (Bilbao y Vitoria)

CiU: $\quad 1 \quad$ (Tarragona)

IU: $\quad 2$ (Córdoba y Guadalajara)

PAR: $\quad 1 \quad$ (Teruel)

PA: $\quad 1 \quad$ (Sevilla)

PFPC: $1 \quad$ (Ceuta)

ATI: $\quad 1 \quad$ (Tenerife)

CDS: $\quad 1 \quad$ (La Palmas)

EI PSOE ha contado siempre con el apoyo de IU, en algunas ocasiones del CDS, y de EU y PNV. EI PP ha sido apoyado por partidos regionalistas (AM, PA, PAR, UM, UPCA, UV, CNG) y, en algunas ocasiones, por el CDS. 


\section{TRASCENDENCIA DE LAS ELECCIONES AUTONOMICAS EN LA COMPOSICIÓN DEL SENADO}

El artículo 69,5 de la Constitución establece que «Las Comunidades Autónomas designarán además un Senador y otro más por cada millón de habitantes de su respectivo territorio. La designación corresponderá a las Asambleas legislativas o, en su defecto, al órgano colegiado superior de la Comunidad Autónoma, de acuerdo con lo que establezcan los Estatutos, que asegurarán, en todo caso, la adecuada representación proporcional».

En este momento las distintas fuerzas parlamentarias de esta Cámara han llegado a un acuerdo a fin de convertir al Senado en auténtica Cámara de representación territorial a través de la reforma de su Reglamento; por ello, el papel de los Senadores autonómicos adquiere especial relevancia en este intento de lograr que la Cámara Alta sea el foro de encuentro entre el Estado y las Comunidades Autónomas ${ }^{24}$.

Tras estas elecciones ${ }^{25}$, los resultados consolidan la mayoria absoluta que ya disfrutaba el PSOE; pasa de 128 senadores - mayoría absoluta- a 129, habiendo logrado este escaño en Cantabria que procede del PP. EI PP aumenta igualmente su número de representantes, de 90 a 93 , lográndolos en Castilla-León, Baleares y Navarra, correspondiendo a los que pierde el CDS; a su vez adquiere un senador más en Murcia - por aumento de la población-que compensará el perdido en Cantabria.

El CDS disminuye de forma espectacular su número de escaños, de siete a uno, perdiendo los correspondientes a la Comunidad de Baleares, Canarias, Castilla-León, Madrid, Navarra y Valencia; sólo conserva, por tanto, el obtenido en las elecciones legislativas de 1989; queda, pues, sin grupo parlamentario. EI PNV - con seis senadores- y CIU - con 12- mantienen el número que ya tenian al no haberse celebrado elecciones autonómicas, en esta fecha, en sus territorios. El Grupo Mixto, finalmente, aumenta el número de senadores de 11 a 15 , habiendo obtenido un representante más, respectivamente, IU, ATI-AIC y Unión Valenciana y recibiendo al único senador del CDS.

Por tanto, pese al mantenimiento de la mayoría absoluta, se observa una consolidación, al alza, de las posiciones de cada grupo, y la desaparición práctica del CDS que quedará reducido a una representación simbólica que le priva de ser una posible alianza para cualquier otra forma política,

24 En este sentido, ver F. FeRnánDEZ-Miranda Alonso: "Los problemas de la distribución territorial del poder en España. La reforma del Senado», RDP, núm. 34, UNED, 1991.

25 Ver El País, de 28 de mayo de 1991. 
papel que jugó durante la última etapa y que, parece ser, le llevó al desastre electoral; en esta segunda transición que parece anunciarse, con el punto de vista puesto en el Senado como foro adecuado para el pacto autonómi$\mathrm{co}$, los hombres del artífice de la primera, quedan absolutamente aislados.

\section{CONCLUSIONES}

Tras el análisis de las elecciones municipales y autonómicas de 26 de mayo de 1991, se puede llegar a las siguientes conclusiones:

a) Se observa una tendencia del electorado hacia posiciones de centro y mayor sensibilidad respecto a la eficacia en la gestión que hacia planteamientos dogmáticos.

b) EI PSOE sigue siendo el partido más votado aunque con un moderado descenso, precisamente entre el electorado mejor informado; compite bien con los partidos nacionalistas tanto en el País Vasco como en Cataluña. EI PP sube, también moderadamente, y logra algunas victorias importantes en ciudades clave. IU obtiene representación en casi todas las instituciones y, a la contra, el CDS desaparece prácticamente.

c) Estas elecciones han tenido una importancia superior a la que corresponde a este tipo de consultas; muestra de ello ha sido la dimisión de Adolfo Suárez, como presidente del CDS, nada más conocer los desastrosos resultados de su partido.

Ello fue debido a la coincidencia de varias circunstancias de gran importancia política: las acusaciones de corrupción con motivo de la oscura financiación de los partidos políticos - débiles económicamente-, con un número muy bajo de militantes, una fuerte dependencia de las subvenciones estatales y sometidos a continuas y costosas campañas electorales; la desaparición de los regímenes del Este europeo y la consiguiente devaluación de las ideologías de izquierda radical; finalmente, la fecha del 92 como año clave, internacionalmente, para España, debiendo elegirse en estos comicios los gestores municipales y autonómicos de estos acontecimientos.

d) Los resultados electorales arrojan un número importante de mayorias minoritarias, lo que hace necesarios los pactos posteriores y la consiguiente exigencia de consenso político. 
e) Estos pactos, en general, han servido para reforzar al partido más votado pero sin mayoría absoluta, buscando la estabilidad en las instituciones; ahora bien, han existido excepciones, tanto en el sentido de gobiernos - bien autonómicos o municipales- de partidos distintos al más votado o alianzas generadoras de crisis políticas.

f) La existencia, tras los pactos, de un gran número de mayorias justas o mínimas favorecen la permanencia de aquél y la consiguiente estabilidad ${ }^{26}$. En minoría no existe ningún gobierno autonómico y sí un número muy reducido de alcaldes, con riesgo cierto de crisis políticas.

Puede decirse que, tras los pactos, las elecciones de 1991 han generado un alto grado de estabilidad política.

g) Pese a que el PP ha subido considerablemente en número de votos, el PSOE ha logrado más mayorias absolutas y su acuerdo puntual con IU le ha posibilitado el acceso a mayor número de instituciones; el apoyo de IU al PSOE no ha exigido un pacto global ni tampoco, en la mayoría de los casos, participación en los cargos, evitando de esta forma corresponsabilizarse con la política del partido gobernante, y manteniendo su papel de oposición; la finalidad fundamental fue evitar gobiernos de derecha. Los resultados electorales logrados por ambos partidos manifiestan la opción mayoritaria de izquierda por parte del electorado.

h) La tendencia señalada se verá compensada por los pactos del PP con numerosos partidos regionalistas moderados; ahora bien, su capacidad de negociación ha sido menor que la del PSOE, siendo lo habitual en sus alianzas las contraprestaciones y la corresponsabilidad. El único pacto global existente ha sido el realizado en Euskadi entre el PNV y el PSOE.

i) Por último, se observan dos correctivos importantes a la tendencia del electorado: el alto indice de abstención y la pérdida para el PSOE de alcaldias en ciudades clave: Madrid, Sevilla, Valencia.

${ }^{26}$ Ver gráfica aportada por el Equipo de Análisis Político de la Universidad Autónoma de Barcelona. Cit. en nota 15. 